\title{
Open clusters as key tracers of Galactic chemical evolution
}

\section{Element abundances in Berkeley 20, Berkeley 29, Collinder 261, and Melotte $66^{\star}, \star \star$}

\author{
P. Sestito ${ }^{1}$, A. Bragaglia ${ }^{2}$, S. Randich ${ }^{1}$, R. Pallavicini ${ }^{3}$, S. M. Andrievsky ${ }^{4,5}$, and S. A. Korotin ${ }^{4,5}$ \\ 1 INAF-Osservatorio Astrofisico di Arcetri, Largo E. Fermi 5, 50125 Firenze, Italy \\ e-mail: [sestito;randich]@arcetri.astro.it \\ 2 INAF-Osservatorio Astronomico di Bologna, via C. Ranzani 1, 40127 Bologna, Italy \\ e-mail: angela.bragaglia@oabo.astro.it \\ 3 INAF-Osservatorio Astronomico "G.S. Vaiana" di Palermo, Piazza del Parlamento 1, 90134 Palermo, Italy \\ e-mail: pallavicini@inaf.it \\ ${ }^{4}$ Department of Astronomy and Astronomical Observatory, Odessa National University, Shevchenko Park, 65014 Odessa, Ukraine \\ e-mail: scan@deneb1.odessa.ua \\ 5 Isaac Newton Institute of Chile, Odessa branch, Ukraine \\ Received 25 February 2008 / Accepted 1 July 2008
}

\section{ABSTRACT}

\begin{abstract}
Context. Galactic open clusters are since long recognized as one of the best tools for investigating the radial distribution of iron and other metals.

Aims. We employed FLAMES at VLT to collect UVES spectra of bright giant stars in a large sample of open clusters, spanning a wide range of Galactocentric distances, ages, and metallicities. We present here the results for four clusters: Berkeley 20 and Berkeley 29, the two most distant clusters in the sample; Collinder 261, the oldest and the one with the minimum Galactocentric distance; Melotte 66

Methods. Equivalent width analysis was carried out using the spectral code MOOG and Kurucz model atmospheres to derive abundances of $\mathrm{Fe}, \mathrm{Al}, \mathrm{Mg}, \mathrm{Si}, \mathrm{Ca}, \mathrm{Ti}, \mathrm{Cr}, \mathrm{Ni}, \mathrm{Ba}$; non-LTE Na abundances were derived by direct line-profile fitting.

Results. We obtain subsolar metallicities for the two anticenter clusters $\mathrm{Be} 20([\mathrm{Fe} / \mathrm{H}]=-0.30, \mathrm{rms}=0.02)$ and Be $29([\mathrm{Fe} / \mathrm{H}]=-0.31$, $\mathrm{rms}=0.03)$, and for Mel $66([\mathrm{Fe} / \mathrm{H}]=-0.33, \mathrm{rms}=0.03)$, located in the third Galactic quadrant, while Cr 261, located toward the Galactic center, has higher metallicity $([\mathrm{Fe} / \mathrm{H}]=+0.13, \mathrm{rms}=0.05 \mathrm{dex})$. The $\alpha$-elements $\mathrm{Si}, \mathrm{Ca}$ and $\mathrm{Ti}$, and the Fe-peak elements $\mathrm{Cr}$ and $\mathrm{Ni}$ are in general close to solar; the $s$-process element $\mathrm{Ba}$ is enhanced. Non-LTE computations of Na abundances indicate solar scaled values, suggesting that the enhancement in Na previously determined in giants in open clusters could be due to neglected non-LTE effects.

Conclusions. Our results support the presence of a steep negative slope of the Fe radial gradient up to about 10-11 kpc from the Galactic center, while in the outer disk the $[\mathrm{Fe} / \mathrm{H}]$ distribution seems flat. All the elemental ratios measured are in very good agreement with those found for disk stars of similar metallicity and no trend with Galactocentric distance seems to be present.
\end{abstract}

Key words. stars: abundances - stars: evolution - Galaxy: disk - Galaxy: open clusters and associations: general Galaxy: open clusters and associations: individual: Berkeley 20, Berkeley 29, Collinder 261, Melotte 66

\section{Introduction}

The radial metallicity gradient - i.e. the behaviour of $[\mathrm{Fe} / \mathrm{H}]$ with Galactocentric radius $\left(R_{\mathrm{gc}}\right)$ - provides a powerful observational constraint on models of Galactic disk formation and chemical evolution (e.g., Tosi 1996; Twarog et al. 1997; Freeman \& Bland-Hawthorn 2002). At the same time, the ratios of the abundances of different elements to Fe, are fundamental for understanding the role of stars with different masses in the Galactic chemical enrichment, and thus to get insights on the initial mass function (IMF) at the epoch of disk formation.

Open clusters are widely recognized as ideal targets for the investigation of chemical abundances and their evolution, due to their internal homogeneity in age and composition, to the

\footnotetext{
* Based on observations collected at ESO telescopes under GTO programmes 71.D-0065, 072.D-0019, and GO programme 076.D-0220.

$\star \star$ Tables 4-7 are only available in electronic form at the CDS via anonymous ftp to cdsarc.u-strasbg.fr $(130.79 .128 .5)$ or via http://cdsweb.u-strasbg.fr/cgi-bin/qcat?J/A+A/488/943
}

fact that their distance is in principle easy to measure, and to their broad coverage of ages and Galactocentric distances. For this reason, many spectroscopic determinations of abundances in open clusters have been carried out during the last years. Among them we cite those based on low resolution spectroscopy by Friel (1995) and Friel et al. (2002) which focused on metallicity $([\mathrm{Fe} / \mathrm{H}])$, and those based on high resolution, by Friel et al. (2003, 2005) Carretta et al. (2004, 2005), Carraro et al. (2004, 2006b, 2007), Yong et al. (2005), most of which present the analysis of several other elements besides iron.

In spite of these observational efforts, various problems/discrepancies persist, especially regarding the abundance gradients. For example, a consensus on the shape of the gradient has not been reached yet. Early works suggested an unique negative slope (e.g., Friel 1995; Carraro et al. 1998; Friel et al. 2002); but Twarog et al. (1997) and Corder \& Twarog (2001) favor a step-like distribution of the Fe content with Galactocentric distance. Very recent results (e.g., Carraro et al. 2004, 2007; 
Yong et al. 2005) suggest that the gradient might become flat for $R_{\mathrm{gc}}$ larger than about $14 \mathrm{kpc}$.

In this context we have carried out two VLT/FLAMES programs, aimed at deriving the gradient, based on the homogeneous analysis of a large sample of open clusters. With the fiber link to UVES we observed giant stars in order to obtain accurate element abundances based on high resolution data. The first program was based on Guaranteed Time Observations (GTO; Pallavicini et al. 2006) and included three open clusters, while within the other one (Randich et al. 2005) nine clusters were observed. The targets span a wide range of ages (from $\sim 1$ Gyr up to $\sim 7 \mathrm{Gyr})$, metallicities $([\mathrm{Fe} / \mathrm{H}]$ from $\sim-0.5$ up to $\sim+0.40)$ and Galactocentric radii $(\sim 6-22 \mathrm{kpc})$.

In Sestito et al. (2006 - hereafter Paper I) and Bragaglia et al. (2008 - hereafter Paper II), the analysis of Fe and other elements in five of the observed clusters (NGC 2660, NGC 2324, NGC 2477, NGC 3960, and Be 32) was presented; in Sestito et al. (2007 - hereafter S07) we reported on abundances in the very metal rich cluster NGC 6253. In this paper we present the analysis of the two far, old, anticenter clusters in the sample by Randich et al. (2005; Berkeley 20, and Berkeley 29), and of two old clusters observed within the GTO program (Cr 261 and Mel 66; Pallavicini et al. 2006). Be 20 and Be 29 are the most distant clusters in the sample, while $\mathrm{Cr} 261$ is the one with the lowest $R_{\mathrm{gc}}$. For all these clusters there are already papers based on high resolution spectra (see next section), but we improve on past studies both on statistics (for most of them only two stars were analyzed) and $\mathrm{S} / \mathrm{N}$ ratio.

The paper is organized as follows: in Sect. 2 we describe the observational samples, data reduction, and abundance analysis; the results are reported in Sect. 3 and discussed in Sect. 4; a summary and conclusion are given in Sect. 5.

\section{Observations and analysis}

\subsection{Sample clusters}

Be 29 - This is the most distant cluster in our sample (actually, the most distant known open cluster) and one of the most metal poor ones. The first photometric study of this cluster is the CCD BVI survey by Kaluzny (1994) who estimated an age of 4 Gyr and a distance of $10.5 \mathrm{kpc}$. Tosi et al. (2004) in a CCD BVI study found, depending on the adopted stellar models, an age of 3.4 or $3.7 \mathrm{Gyr},(m-M)_{0}=15.6$ or 15.8 (implying $R_{\mathrm{gc}}=21$ or $\left.22 \mathrm{kpc}\right)$, with $E(B-V)=0.13$ or 0.10 , and metallicity lower than solar $(Z=0.006$ or 0.004$)$, depending on the adopted stellar models. These values were confirmed by Bragaglia et al. (2005), who presented radial velocity measurements for red giant branch (RGB) stars, and by Bragaglia $\&$ Tosi (2006) who give the following parameters based on the Padova evolutionary tracks: age $\sim 3.7 \mathrm{Gyr},(m-M)_{0}=15.6$, $E(B-V)=0.12, Z=0.004$, distance $13.05 \mathrm{kpc}$.

Spectroscopic investigations of Be 29 were presented by Carraro et al. (2004), Yong et al. (2005), and Frinchaboy et al. (2006). Carraro et al. and Yong et al. derived a metallicity $[\mathrm{Fe} / \mathrm{H}]=-0.44$ (for two red clump stars) and -0.54 (for two stars near the RGB tip), respectively. They also presented results for abundances of several elements in addition to Fe. Frinchaboy et al. investigated the radial velocity of the cluster, concluding that its properties are in agreement with the Galactic anticenter stellar structure, also known as the Monoceros Ring.

Be 20 - Also Be 20 is a very distant cluster, with a distance of $8.4 \mathrm{kpc}$ from the Sun and $R_{\mathrm{gc}}$ of about $16 \mathrm{kpc}$. The first photometric study of Be 20 is the CCD VI survey by MacMinn et al. (1994); they estimated an age of 5-6 Gyr, a reddening $E(V-I)=0.16$, a metallicity $[\mathrm{Fe} / \mathrm{H}]=-0.23$ and $(m-M)_{V}=15.0$; their data were used for the present paper. Recently Andreuzzi et al. $(2007,2008)$ presented a new photometry, obtaining results in very good agreement with MacMinn et al. (1994).

Two spectroscopic investigations of Be 20 were carried out so far. Friel et al. (2002), on the basis of low resolution spectra of 6 stars, derived an average $[\mathrm{Fe} / \mathrm{H}]$ value of $-0.61 \pm 0.14$ dex. Yong et al. (2005) derived $[\mathrm{Fe} / \mathrm{H}]=-0.45$ and -0.53 for two stars near the RGB tip observed at high resolution.

Cr 261 - This is one of the oldest open clusters in the Galaxy, with an age in the range 6-10 Gyr (but the upper value is most probably spurious, see the discussion in Bragaglia \& Tosi 2006). It is also one of the few known old open clusters inside the Solar circle $\left(R_{\mathrm{gc}} \sim 7.5 \mathrm{kpc}\right)$. Photometric studies of the cluster were carried out by Janes \& Phelps (1994), Mazur et al. (1995), and Gozzoli et al. (1996); not surprisingly, given the low Galactic latitude and the direction toward the center, they all agree on a high reddening values, ranging from $E(B-V)=0.22$ up to 0.34 (depending on the assumed metallicity). The Gozzoli et al. results have been recently revised by Bragaglia \& Tosi (2006), who find a best fit solution for solar metallicity, distance modulus $(m-M)_{0}=12.2, E(B-V)=0.30$, and age 6 Gyr.

The metallicity of $\mathrm{Cr} 261$ has been measured by means of low resolution spectroscopy by Friel et al. (2002): from the analysis of 21 giants they derived $[\mathrm{Fe} / \mathrm{H}]=-0.16 \pm 0.13$. In a more recent study the same group determined the metallicity from high resolution data of four giants, obtaining $[\mathrm{Fe} / \mathrm{H}]=-0.22 \pm 0.05$ (Friel et al. 2003). A higher metal content is reported by Carretta et al. (2005), who derived $[\mathrm{Fe} / \mathrm{H}]=-0.03 \pm 0.03$ (i.e. solar metallicity) and proposed as probable reasons for the different result differences in the model atmospheres, in the spectral resolution and equivalent width $(E W)$ measurements, and in the atomic parameters (oscillator strength).

Mel 66 - Deep CCD photometry for Mel 66 was provided by Kassis et al. (1997), who quote an age of $4 \pm 1 \mathrm{Gyr}$ and $(m-M)_{0}=13.2$, with $[\mathrm{Fe} / \mathrm{H}]=-0.51$ and $E(B-V)$ from 0.14 to 0.21 (from isochrone fitting). Combining the distance modulus with the Galactic coordinates, an $R_{\mathrm{gc}}$ of about $10 \mathrm{kpc}$ is obtained.

Previous studies of the metallicity of the cluster are those by Twarog et al. (1995) who derived $[\mathrm{Fe} / \mathrm{H}]=-0.39$ from $U B V$ photometry of TO stars, and by Friel et al. (2002) who obtained $[\mathrm{Fe} / \mathrm{H}]=-0.47 \pm 0.09$ from low resolution spectroscopy of four cluster giants. The only high resolution spectroscopic investigation carried out so far for Mel 66 is by Gratton \& Contarini (1994), who studied two giants and obtained $[\mathrm{Fe} / \mathrm{H}]=-0.38 \pm 0.15$.

The properties of the target clusters are summarized in Table 1: references for ages, distance moduli and reddening are also given; the Galactocentric radii were taken from the collections by Friel et al. (2002) and Friel (2006); slightly different values from other literature sources were mentioned above. The $[\mathrm{Fe} / \mathrm{H}]$ values cover the whole range found in literaure (see above). 
Table 1. Target clusters and their properties.

\begin{tabular}{lrrlllllll}
\hline \hline Cluster & $l$ & $b$ & $\begin{array}{l}\text { Age } \\
(\mathrm{Gyr})\end{array}$ & $\begin{array}{c}{[\mathrm{Fe} / \mathrm{H}]} \\
(\mathrm{lit} .)\end{array}$ & $\begin{array}{l}R_{\mathrm{gc}} \\
(\mathrm{kpc})\end{array}$ & $\begin{array}{l}(m-M)_{0} \\
(\mathrm{mag})\end{array}$ & $\begin{array}{l}E(B-V) \\
(\mathrm{mag})\end{array}$ & $\begin{array}{l}E(V-I) \\
(\mathrm{mag})\end{array}$ & $\begin{array}{l}\text { Reference } \\
\text { (age, dist.mod., reddening) }\end{array}$ \\
\hline Be 20 & 203.5 & -17.4 & $5-6$ & $-0.5 \rightarrow-0.2$ & 16.4 & 14.6 & - & 0.16 & MacMinn et al. (1994) \\
Be 29 & 198.0 & 8.0 & $3-4$ & $-0.7 \rightarrow-0.4$ & 22.0 & 15.6 & 0.12 & - & Bragaglia \& Tosi (2006) \\
Mel 66 & 260.5 & -14.2 & $3-4$ & $-0.5 \rightarrow-0.4$ & 10.2 & 13.2 & $0.14-0.21$ & - & Kassis et al. (1997) \\
Cr 261 & 301.7 & -5.5 & $6-7$ & $-0.2 \rightarrow 0.0$ & 7.5 & 12.2 & 0.30 & - & Bragaglia \& Tosi (2006) \\
\hline
\end{tabular}

\subsection{Observations and data reduction}

The target clusters were observed with the multi-object instrument FLAMES (Pasquini et al. 2000) on VLT/UT2 (ESO, Chile). The fiber link to UVES was used to obtain highresolution spectra $(R=45000)$ for RGB and red clump objects.

For all the clusters we performed observations with the CD3 cross-disperser, covering the wavelength range 4750-6800 А; for Cr 261 and Mel 66 we also obtained spectra using the CD4 grating ( 6600-10600 $\AA$ ). The observations of Be 20 and $\mathrm{Be} 29$ were carried out in service mode during the period February-March 2006, while Cr $261 \mathrm{Mel} 66$ and were observed in May and December 2003. A log of observations (date, UT, exposure time, grating configuration, number of stars) is given in Table 2. The spectra were reduced using the dedicated pipeline, and we analyzed the 1-d, wavelength-calibrated spectra using standard $\operatorname{IRAF}^{1}$ packages. Radial velocities $(R V)$ were derived with the task RVIDLINES in IRAF; corrections for the contribution of telluric lines were performed using TELLURIC and individual exposures were summed (see Papers I and II for more details).

To maintain the highest homogeneity in our work we tried to select always the same kind of stars, i.e., red clump stars. For the present observations we selected seven stars at the red clump of Mel 66, five stars at the red clump and one star near the RGB tip in Be 29, six stars at the clump and one slightly brighter in $\mathrm{Cr} 261$, and six stars on the RGB near the clump level for Be 20 (since this cluster has no well defined clump). Figures 1 and 2 show examples the spectra of all the stars observed, in a small wavelength region.

We report information on the stars of the four clusters in Table 3: we give the identification, equatorial coordinates (J2000), magnitudes (Cols. 4-7), number of exposures for each star, the average heliocentric radial velocity $(R V)$ and its rms, the $S / N$ measured in the two wavelength regions around $\sim 5600$ and $6300 \AA$, and a note (M stands for "member", NM for "non member", NM? for "doubtful member"). The $B, V$, and $I$ magnitudes come from the different sources referenced below for each cluster, the $K$ magnitudes are taken from 2MASS (Skrutskie et al. 2006) $)^{2}$.

For Be 20 we adopted the identification number used for the FLAMES pointings, based on the EIS catalogue used to select and point stars. Only two stars of the six observed turned out to be secure cluster members. These stars, 1201 and 1240, have $R V$ of about $78.5 \mathrm{~km} \mathrm{~s}^{-1}$, that agrees with the average radial

${ }^{1}$ IRAF is distributed by the National Optical Astronomical Observatories, which are operated by the Association of Universities for Research in Astronomy, under contract with the National Science Foundation.

2 The Two Micron All Sky Survey is a joint project of the University of Massachusetts and the Infrared Processing and Analysis Center/California Institute of technology, funded by the national Aeronautics and Space Administration and the National Science Foundation.
Table 2. Log of the observations.

\begin{tabular}{cccccc}
\hline \hline Cluster & $\begin{array}{c}\text { Date } \\
\text { yyyy-mm-dd }\end{array}$ & $\begin{array}{c}\text { UT beginning } \\
\text { hh:mm:ss }\end{array}$ & $\begin{array}{c}\text { Exptime } \\
\text { (s) }\end{array}$ & CD & $\begin{array}{c}\text { No. of } \\
\text { stars }\end{array}$ \\
\hline Be 20 & $2006-02-09$ & $03: 07: 30$ & 2775 & CD3 & 6 \\
& $2006-02-11$ & $01: 19: 26$ & 2775 & CD3 & 6 \\
& $2006-02-11$ & $02: 28: 10$ & 2775 & CD3 & 6 \\
& $2006-02-11$ & $03: 23: 46$ & 2775 & CD3 & 6 \\
& $2006-02-11$ & $04: 21: 48$ & 2775 & CD3 & 6 \\
& $2006-02-12$ & $01: 46: 45$ & 2775 & CD3 & 6 \\
Be 29 & $2006-02-12$ & $02: 45: 26$ & 2775 & CD3 & 6 \\
& $2006-02-12$ & $03: 54: 09$ & 2775 & CD3 & 6 \\
& $2006-02-13$ & $00: 36: 51$ & 2775 & CD3 & 6 \\
& $2006-02-13$ & $01: 28: 16$ & 2775 & CD3 & 6 \\
& $2006-02-13$ & $02: 45: 03$ & 2775 & CD3 & 6 \\
& $2006-02-13$ & $03: 35: 43$ & 2775 & CD3 & 6 \\
& $2006-02-18$ & $02: 29: 11$ & 2775 & CD3 & 6 \\
& $2006-03-06$ & $00: 32: 12$ & 2775 & CD3 & 6 \\
Mel 66 & $2003-12-01$ & $06: 27: 38$ & 3000 & CD3 & 7 \\
& $2003-12-01$ & $07: 24: 40$ & 3359 & CD3 & 7 \\
& $2003-12-01$ & $08: 22: 14$ & 2188 & CD3 & 7 \\
& $2003-12-02$ & $05: 25: 40$ & 3600 & CD4 & 7 \\
& $2003-12-02$ & $06: 28: 43$ & 3600 & CD4 & 7 \\
Cr 261 61 (2) & $2003-12-02$ & $07: 29: 46$ & 3600 & CD4 & 7 \\
& $2003-05-27$ & $23: 11: 47$ & 1200 & CD3 & 7 \\
& $2003-05-27$ & $23: 34: 38$ & 3600 & CD3 & 7 \\
& $2003-05-28$ & $00: 37: 16$ & 3600 & CD3 & 7 \\
& $2003-05-28$ & $01: 39: 51$ & 4090 & CD3 & 7 \\
& $2003-05-28$ & $03: 02: 50$ & 3600 & CD4 & 7 \\
& $2003-05-28$ & $04: 06: 38$ & 3600 & CD4 & 7 \\
\hline
\end{tabular}

velocity found by Yong et al. $\left(2005:+78.9, \sigma=0.7 \mathrm{~km} \mathrm{~s}^{-1}\right)$, therefore we label the two objects as cluster members; furthermore, 1201 and 1240 are the two stars closest to the cluster center. Star 1240 has also been observed by Yong et al. (their star 8, as seen from their Table 2). Stars 1401 and 1716 are clearly $R V$ non members; the remaining objects, 1519 and 1666, have $R V$ differing by about $6-7 \mathrm{~km} \mathrm{~s}^{-1}$ from those of members. Given the spectral resolution of our data, we can derive $R V$ with a precision of better than $1 \mathrm{~km} \mathrm{~s}^{-1}$, so we conclude that 1519 and 1666 are most probably non members of Be 20 . Another possibility is that they are long period binaries, but our data were obtained on too short a baseline to verify this possibility. Magnitudes in the Johnson-Cousins system were taken from our reference photometry (MacMinn et al. 1994) for the two member stars. For stars 1666 and 1716 we used the $V I_{\mathrm{C}}$ values from Andreuzzi et al. (2008), while for the remaining two objects we give in Table 3 the EIS magnitude, shifted to the MacMinn et al. and Kassis et al. systems, respectively.

For Be 29 we selected five secure cluster members, as deduced from the $R V$ s measured by Bragaglia et al. (2005) and Carraro et al. (2004: our star 398, their 801). Also the sixth target (star 602), chosen among the red clump ones, turned out to be a member, as shown by the very similar $R V$. The average $R V$ is $24.66 \pm 0.37 \mathrm{~km} \mathrm{~s}^{-1}$. The identifications and $B V I_{\mathrm{C}}$ magnitudes 


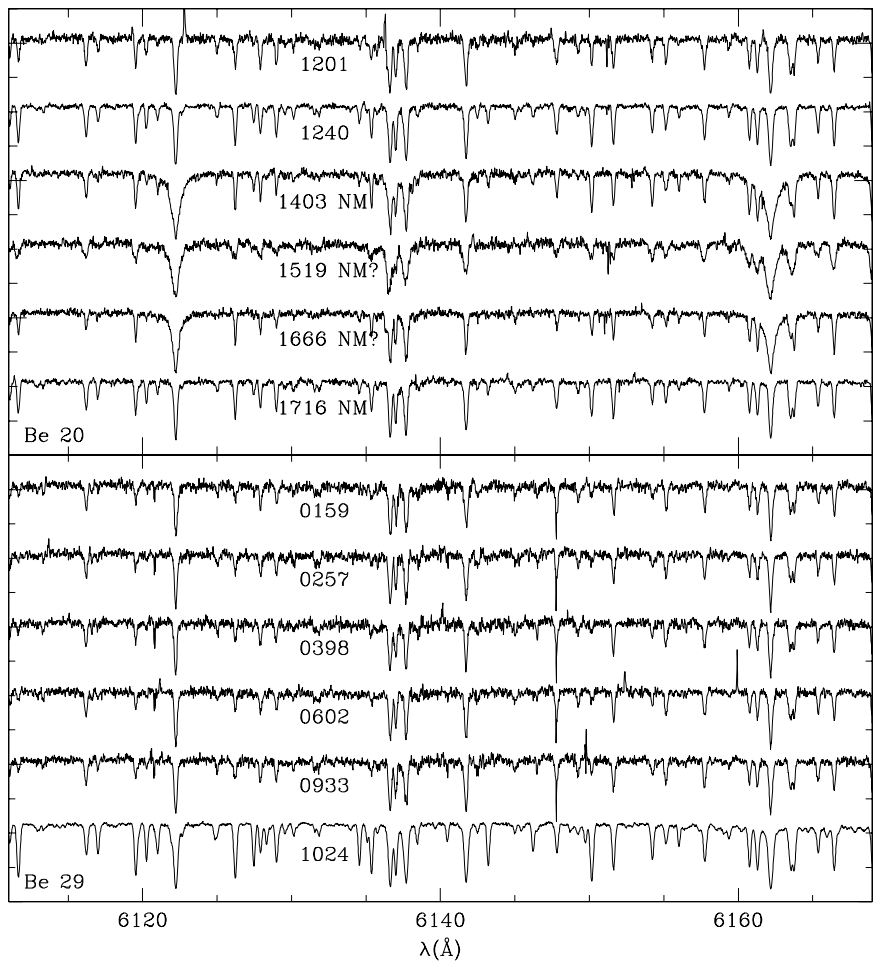

Fig. 1. A region of the spectra of stars in Be 20 and Be 29.

in Table 3 are taken from the photometry by Tosi et al. (2004), which is also in perfect agreement with Kaluzny (1994).

The stars observed in Cr 261 share very similar $R V \mathrm{~s}$ and therefore all of them are considered members (the average $R V$ is $-25.43 \pm 1.11 \mathrm{~km} \mathrm{~s}^{-1}$ ). The ID adopted is the provisional one used in the FLAMES pointings, coming from the catalogue based on WFI@2.2 m data. Estimates of the reddening of Cr 261 are very uncertain, with values spanning from $E(B-V)=0.22$ up to 0.34 (see Spanò 2005). Recent studies (e.g. Carretta et al. 2005 ) seem to indicate a value close to 0.30 , at least in the central region. We adopt here the WFI $B V I$ photometry calibrated by Prisinzano (private communication).

As for as Mel 66, five of the observed stars have $R V$ around $21 \mathrm{~km} \mathrm{~s}^{-1}$ (the mean $R V$ is $+21.25 \pm 0.37 \mathrm{~km} \mathrm{~s}^{-1}$ ); star 1865 has $R V=+17.85 \mathrm{~km} \mathrm{~s}^{-1}$ and is therefore labeled as a doubtful member. Star 1614 rotates very rapidly and was discarded from further analysis. For this cluster we adopted the $V I_{\mathrm{C}}$ photometry by Kassis et al. (1997).

\subsection{Abundance analysis}

The method of analysis is described in Papers I and II; therefore we provide here only a brief summary, and we refer the reader to those papers for more complete information on the line lists adopted, references for atomic parameters and damping. First, we determined the solar abundances of $\mathrm{Fe}$ and other elements, in order to fix the zero points of the abundance scale and to minimize errors in the results. For the Sun we obtain $\log n(\mathrm{Fe} \mathrm{I})=7.49 \pm 0.04$ (standard deviation, or $\mathrm{rms}$ ) and $\log n(\mathrm{Fe}$ II $)=7.54 \pm 0.03$, adopting the following effective temperature, surface gravity and microturbulence velocity: $T_{\text {eff }}=5779 \mathrm{~K} \log g=4.44$, and $\xi=0.8 \mathrm{~km} \mathrm{~s}^{-1}$; solar abundances of other elements are reported in Table 3 of Paper II.

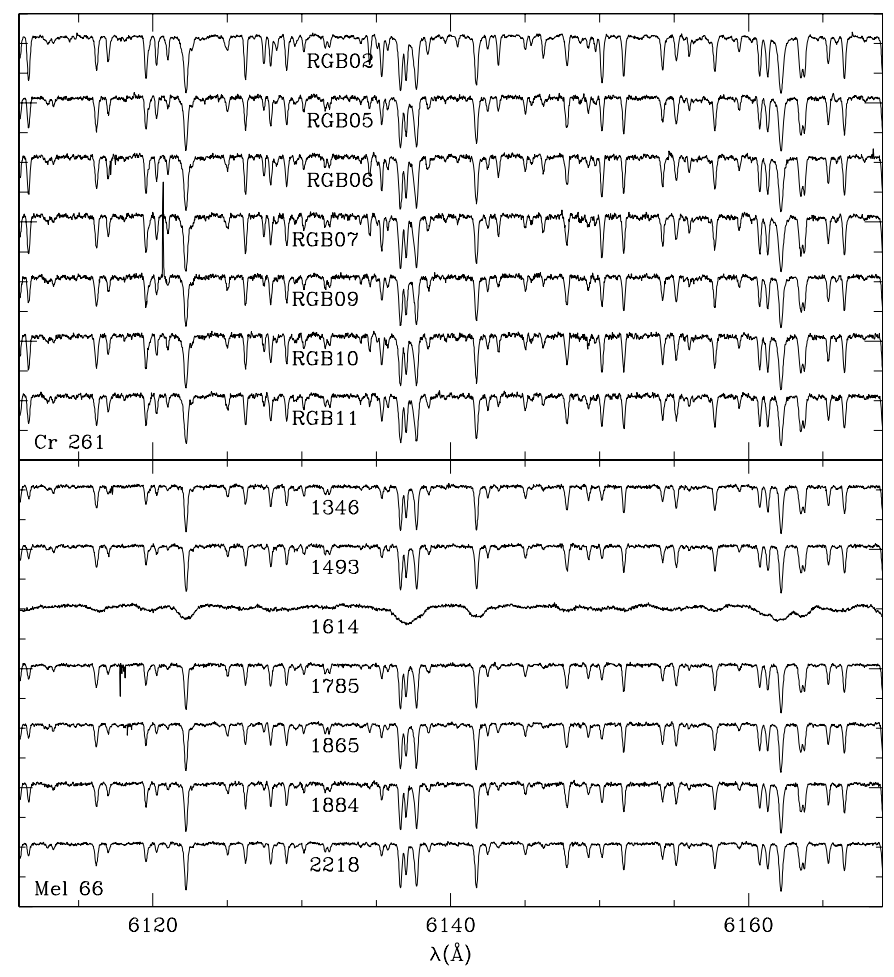

Fig. 2. A region of the spectra of stars in Cr 261 and Mel 66.

\subsubsection{Equivalent widths}

$E W$ analysis was carried out using a recent version (2006) of the code MOOG (Sneden 1973) ${ }^{3}$ and using model atmospheres by Kurucz (1993). Continuum tracing and normalization of the spectra were carried out using CONTINUUM within IRAF, dividing the spectra in small regions (50 $\AA$ ) and visually checking the output. The $E W$ measurements were carried out with the program SPECTRE, developed by Chris Sneden (see Fitzpatrick \& Sneden 1987), which performs a Gaussian fitting of the line profiles. The values are available in electronic Tables $4-7$, where the first two columns list the wavelengths and element, and the others show the corresponding $E W$ for each star. Continuum tracing and $E W$ determination are very critical steps in the analysis, possibly leading to discrepancies among the results of the various authors. These procedures are particularly problematic for giant Population I stars, that suffer for line blending due to the high metal content and the cool temperature. In the case of the two most distant clusters Be 20 and Be 29 the determination of continuum and of $E W \mathrm{~s}$ has been more difficult and uncertain due to the non optimal $S / N$.

\subsubsection{Stellar parameters}

Initial effective temperatures were derived from $B, V, I$, and $K$ photometry, applying the calibration by Alonso et al. (1999). For Be 29 and $\mathrm{Cr} 261$ we used both $B-V$ and $V-K$ colors; for Mel 66 and Be 20 we used $V-K$ and $V-I$, after having converted the Cousin colors by Kassis et al. (1997) and MacMinn et al. (1994), respectively, into the Johnson ones (required in the Alonso calibration) with the formula: $V-I_{\mathrm{J}}=1.285\left(V-I_{\mathrm{C}}\right)$ (Bessell 1979). The $T_{\text {eff }}$ were then optimized during the analysis, in order to eliminate possible trends of $\log n(\mathrm{Fe} \mathrm{I})$ vs. the excitation potential, i.e. to satisfy the excitation equilibrium

\footnotetext{
${ }^{3}$ http://verdi.as.utexas.edu/
} 
Table 3. Stars observed in the four clusters; see text for details.

\begin{tabular}{|c|c|c|c|c|c|c|c|c|c|c|}
\hline $\begin{array}{l}\text { Star } \\
\text { ID }\end{array}$ & RA & $\overline{\mathrm{Dec}}$ & $\overline{B B}$ & 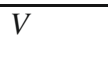 & $\overline{I I}$ & $\overline{\bar{K}}$ & $\begin{array}{l}\text { No. exp. } \\
\text { CD3 }\end{array}$ & $\begin{array}{l}R V(\mathrm{rms}) \\
\left(\mathrm{km} \mathrm{s}^{-1}\right)\end{array}$ & $\bar{S} S / N$ & Notes \\
\hline \multicolumn{11}{|c|}{ Berkeley 20} \\
\hline OC03-001201 & 053236.774 & +001104.84 & & 16.177 & 14.929 & 13.276 & 6 & $78.47(0.62)$ & $35-60$ & M \\
\hline OC03-001240 & 053238.963 & +00 1120.37 & & 15.154 & 13.727 & 11.850 & 6 & $78.58(0.22)$ & $40-80$ & M \\
\hline OC03-001403 & 05325.609 & +001244.04 & & 15.88 & 14.53 & 12.974 & 6 & $-2.95(0.90)$ & $40-80$ & $\mathrm{NM}$ \\
\hline OC03-001519 & 053254.960 & +001407.30 & & 16.32 & 15.09 & 13.653 & 6 & $72.18(0.58)$ & $35-70$ & NM? \\
\hline OC03-001666 & 053246.417 & +001552.19 & & 15.919 & 14.673 & 13.153 & 6 & $85.45(0.51)$ & $30-50$ & NM? \\
\hline OC03-001716 & 053250.057 & +001616.43 & & 15.447 & 13.992 & 12.233 & 6 & $37.67(0.22)$ & $40-80$ & NM \\
\hline \multicolumn{11}{|c|}{ Berkeley 29} \\
\hline 159 & 065301.602 & +165621.11 & 17.625 & 16.627 & 15.574 & 14.266 & 8 & $24.64(0.85)$ & $35-50$ & M \\
\hline 257 & 065304.320 & +165539.37 & 17.578 & 16.608 & 15.548 & 13.508 & 8 & $24.38(1.21)$ & $35-50$ & M \\
\hline 398 & 065308.081 & +165540.27 & 17.535 & 16.591 & 15.524 & 14.227 & 8 & $24.24(1.16)$ & $25-50$ & M \\
\hline 602 & 065255.488 & +165739.30 & 17.573 & 16.579 & 15.469 & 14.119 & 8 & $24.84(0.57)$ & $25-50$ & M \\
\hline 933 & 065304.486 & +165744.69 & 17.435 & 16.447 & 15.322 & 13.932 & 8 & $25.27(1.03)$ & $40-50$ & M \\
\hline 1024 & 065307.133 & +165712.67 & 16.067 & 14.458 & 12.795 & 10.600 & 8 & $24.59(0.42)$ & $100-180$ & $\mathrm{M}$ \\
\hline \multicolumn{11}{|c|}{ Melotte 66} \\
\hline 1346 & 072617.281 & -474359.89 & & 14.59 & 13.36 & 11.773 & 3 & $21.55(0.34)$ & $80-110$ & M \\
\hline 1493 & 072634.571 & -474247.03 & & 14.68 & 13.39 & 11.823 & 3 & $21.32(0.37)$ & $80-100$ & M \\
\hline 1614 & 072623.682 & -474201.83 & & 14.77 & 13.43 & 11.866 & 3 & & $80-100$ & fast rotator \\
\hline 1785 & 072602.491 & -474055.85 & & 14.59 & 13.35 & 11.795 & 3 & $21.60(0.32)$ & $90-110$ & $\mathrm{M}$ \\
\hline 1865 & 072557.923 & -474023.16 & & 14.51 & 13.29 & 11.736 & 3 & $17.85(0.50)$ & $110-130$ & NM? \\
\hline 1884 & 072619.880 & -474015.72 & & 14.71 & 13.52 & 11.767 & 3 & $21.04(0.34)$ & $80-100$ & M \\
\hline 2218 & 072626.646 & -473755.66 & & 14.48 & 13.29 & 11.826 & 3 & $20.72(0.52)$ & $100-115$ & M \\
\hline \multicolumn{11}{|c|}{ Collinder 261} \\
\hline RGB02 & 123734.636 & -682325.32 & 14.384 & 12.937 & 11.345 & 9.184 & 3 & $-25.63(0.32)$ & $100-130$ & M \\
\hline RGB05 & 123812.612 & -682149.49 & 15.480 & 14.139 & 12.635 & 10.020 & 3 & $-27.09(0.16)$ & $70-100$ & M \\
\hline RGB06 & 123807.229 & -682230.82 & 15.380 & 14.011 & 12.447 & 10.343 & 3 & $-24.91(0.23)$ & $80-100$ & M \\
\hline RGB07 & 123755.184 & -682235.82 & 15.394 & 14.013 & 12.436 & 10.318 & 3 & $-25.70(0.27)$ & $60-100$ & M \\
\hline RGB09 & 123812.338 & -682031.46 & 15.548 & 14.212 & 12.700 & 10.684 & 3 & $-25.96(0.27)$ & $80-120$ & M \\
\hline RGB10 & 123753.921 & -682148.61 & 15.675 & 14.368 & 12.873 & 10.767 & 3 & $-25.32(0.07)$ & $60-90$ & M \\
\hline RGB11 & 123759.670 & -682349.60 & 15.438 & 14.145 & 12.654 & 10.651 & 3 & $-23.42(0.18)$ & $80-100$ & M \\
\hline
\end{tabular}

condition. The initial surface gravity was computed from the photometry, with bolometric corrections derived following the prescriptions by Alonso et al. (1999), and with clump masses of $1.20 M_{\odot}$ for Be 20 (age 5-6 Gyr), Be 29 and Mel 66 (ages $\sim 4$ Gyr), $1.15 M_{\odot}$ for Cr 261 (age $\sim 6-7$ Gyr). The final spectroscopic gravities were chosen by imposing the ionization equilibrium condition, i.e. $\log n(\mathrm{Fe}$ II $)-\log n(\mathrm{Fe} \mathrm{I})=0.05$ (as found for the Sun). The initial microturbulence velocity, $\xi$, was computed following the relationship by Carretta et al. (2004): $\xi=[1.5-0.13 \times \log g] \mathrm{km} \mathrm{s}^{-1}$, and then optimized by zeroing the slope of $\log n(\mathrm{Fe} \mathrm{I})$ as a function of theoretically expected $E W \mathrm{~s}$ for a fixed abundance.

\subsubsection{Errors}

Final abundances are affected by random and systematic errors, whose main sources are uncertainties in oscillator strengths and $E W \mathrm{~s}$, and uncertainties in the stellar parameters.

Internal errors due to uncertainties in the oscillator strengths are minimized since our scale is referred to the Sun (see also Paper I). The effect of random errors in $E W \mathrm{~s}$ and in the atomic parameters on the derived abundance for a single star is well represented by $\sigma_{1}$, the standard deviation from the mean abundance based on the whole set of lines (see Table 8). Errors due to uncertainties in $T_{\mathrm{eff}}, \log g$, and $\xi\left(\sigma_{2}\right)$ were estimated following the method by Carretta et al. (2004), where a detailed description of the procedure can be found, while in Paper I we give the values found by us for our clusters. For Cr 261 and Mel 66 the estimated random errors are similar to those in NGC 3960 (Paper I) since the spectra of the two clusters have very similar $S / N$ : $\sim 20 \mathrm{~K}$ for the $T_{\text {eff }}, \sim 0.10$ dex for the gravity and $0.10 \mathrm{~km} \mathrm{~s}^{-1}$ for microturbulence. In the case of $\mathrm{Be} 20$ and $\mathrm{Be} 29$ these random errors are slightly larger, due to the worse $S / N: \sim 50 \mathrm{~K}$ for the $T_{\text {eff }}, \sim 0.2$ dex for the gravity and $0.15 \mathrm{~km} \mathrm{~s}^{-1}$ for microturbulence. Then, we computed the sensitivity of $[\mathrm{Fe} / \mathrm{H}]$ (and in general of $[\mathrm{X} / \mathrm{H}]$ ) to errors in the individual stellar parameters, and we quadratically added the three contributions in order to obtain $\sigma_{2}$ (see Table 13 in Paper I and Table 12 in Paper II).

The final error $\sigma_{\text {tot }}$ for Fe is the quadratic sum of $\sigma_{1}$ and $\sigma_{2}$; for other elements $([\mathrm{X} / \mathrm{Fe}])$, we computed a total error $\sigma_{1 \text { tot }}$ by quadratically adding the $\sigma_{1}$ on $[\mathrm{Fe} / \mathrm{H}]$ and the $\sigma_{1}$ on $[\mathrm{X} / \mathrm{H}]$. The reader can find an estimate of $\sigma_{2}$ also for the abundances $[\mathrm{X} / \mathrm{H}]$ in Table 12 of Paper II. Note that the two stars in Be 20, and star 602 in Be 29 have $\sigma_{1}$ values very similar to those of stars RGB02 and RGB11 in Cr 261, although the spectra of the latter ones have a higher $S / N$. This result might be due to the higher $[\mathrm{Fe} / \mathrm{H}]$ of $\mathrm{Cr} 261$ stars, for which it is more difficult to determine the continuum position during the $E W$ measurement, and which suffer of stronger line blending.

Finally, we checked the presence of systematic errors related to the method of analysis by analyzing two Hyades giants with the same technique (see Paper I). We found abundances in 
Table 8. Stellar parameters and Fe abundances.

\begin{tabular}{|c|c|c|c|c|c|c|c|c|c|}
\hline Star & $\begin{array}{l}T_{\text {eff,phot }} \\
(\mathrm{K})\end{array}$ & $\overline{l o g} g_{\text {phot }}$ & $\begin{array}{l}T_{\text {eff }, \text { spec }} \\
(\mathrm{K})\end{array}$ & $\overline{l o g} g_{\text {spec }}$ & $\begin{array}{l}\xi_{\text {spec }} \\
\left(\mathrm{km} \mathrm{s}^{-1}\right)\end{array}$ & 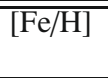 & 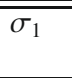 & $\overline{\overline{\sigma_{\text {tot }}}}$ & 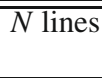 \\
\hline \multicolumn{10}{|c|}{ Berkeley 20} \\
\hline 1201 & 4521 & 2.47 & 4700 & 2.10 & 1.35 & -0.28 & 0.10 & 0.13 & 62 \\
\hline 1240 & 4226 & 1.97 & 4400 & 1.70 & 1.27 & -0.31 & 0.12 & 0.14 & 94 \\
\hline \multicolumn{6}{|l|}{ Average } & \multicolumn{2}{|l|}{-0.30} & \multicolumn{2}{|l|}{0.02} \\
\hline \multicolumn{10}{|c|}{ Berkeley 29} \\
\hline 159 & 4994 & 2.51 & 4994 & 2.70 & 1.17 & -0.28 & 0.14 & 0.16 & 78 \\
\hline 257 & 4962 & 2.49 & 4930 & 2.58 & 1.18 & -0.36 & 0.13 & 0.15 & 76 \\
\hline 398 & 5045 & 2.51 & 5020 & 2.70 & 1.17 & -0.31 & 0.14 & 0.16 & 76 \\
\hline 602 & 4940 & 2.47 & 4970 & 2.35 & 1.28 & -0.34 & 0.11 & 0.14 & 80 \\
\hline 933 & 4915 & 2.40 & 4930 & 2.28 & 1.25 & -0.30 & 0.13 & 0.15 & 78 \\
\hline 1024 & 3954 & 0.95 & 4050 & 1.03 & 1.37 & -0.29 & 0.12 & 0.14 & 79 \\
\hline \multicolumn{6}{|l|}{ Average } & \multicolumn{2}{|l|}{-0.31} & \multicolumn{2}{|l|}{0.03} \\
\hline \multicolumn{10}{|c|}{ Melotte 66} \\
\hline 1346 & 4688 & 2.43 & 4750 & 2.00 & 1.17 & -0.37 & 0.09 & 0.11 & 104 \\
\hline 1493 & 4609 & 2.42 & 4770 & 2.15 & 1.20 & -0.35 & 0.09 & 0.11 & 110 \\
\hline 1785 & 4691 & 2.43 & 4770 & 2.05 & 1.20 & -0.30 & 0.09 & 0.11 & 101 \\
\hline 1865 & 4717 & 2.41 & 4717 & 2.05 & 1.24 & -0.34 & 0.08 & 0.10 & 98 \\
\hline 1884 & 4669 & 2.47 & 4750 & 2.45 & 1.23 & -0.30 & 0.06 & 0.08 & 101 \\
\hline 2218 & 4814 & 2.45 & 4850 & 2.39 & 1.25 & -0.31 & 0.07 & 0.09 & 103 \\
\hline Average & & & & & & -0.33 & & 0.03 & \\
\hline \multicolumn{10}{|c|}{ Collinder 261} \\
\hline RGB02 & 4465 & 2.08 & 4350 & 1.70 & 1.25 & +0.12 & 0.10 & 0.12 & 102 \\
\hline RGB05 & 4450 & 2.48 & 4600 & 2.00 & 1.24 & +0.14 & 0.13 & 0.15 & 114 \\
\hline RGB06 & 4566 & 2.53 & 4500 & 2.30 & 1.18 & +0.16 & 0.09 & 0.11 & 113 \\
\hline RGB07 & 4546 & 2.52 & 4546 & 2.15 & 1.20 & +0.18 & 0.09 & 0.11 & 111 \\
\hline RGB09 & 4652 & 2.66 & 4720 & 2.05 & 1.27 & +0.04 & 0.09 & 0.11 & 120 \\
\hline RGB10 & 4652 & 2.72 & 4700 & 2.35 & 1.20 & +0.20 & 0.09 & 0.11 & 120 \\
\hline RGB11 & 4709 & 2.66 & 4670 & 2.15 & 1.13 & +0.09 & 0.11 & 0.13 & 117 \\
\hline Average & & & & & & +0.13 & & 0.05 & \\
\hline
\end{tabular}
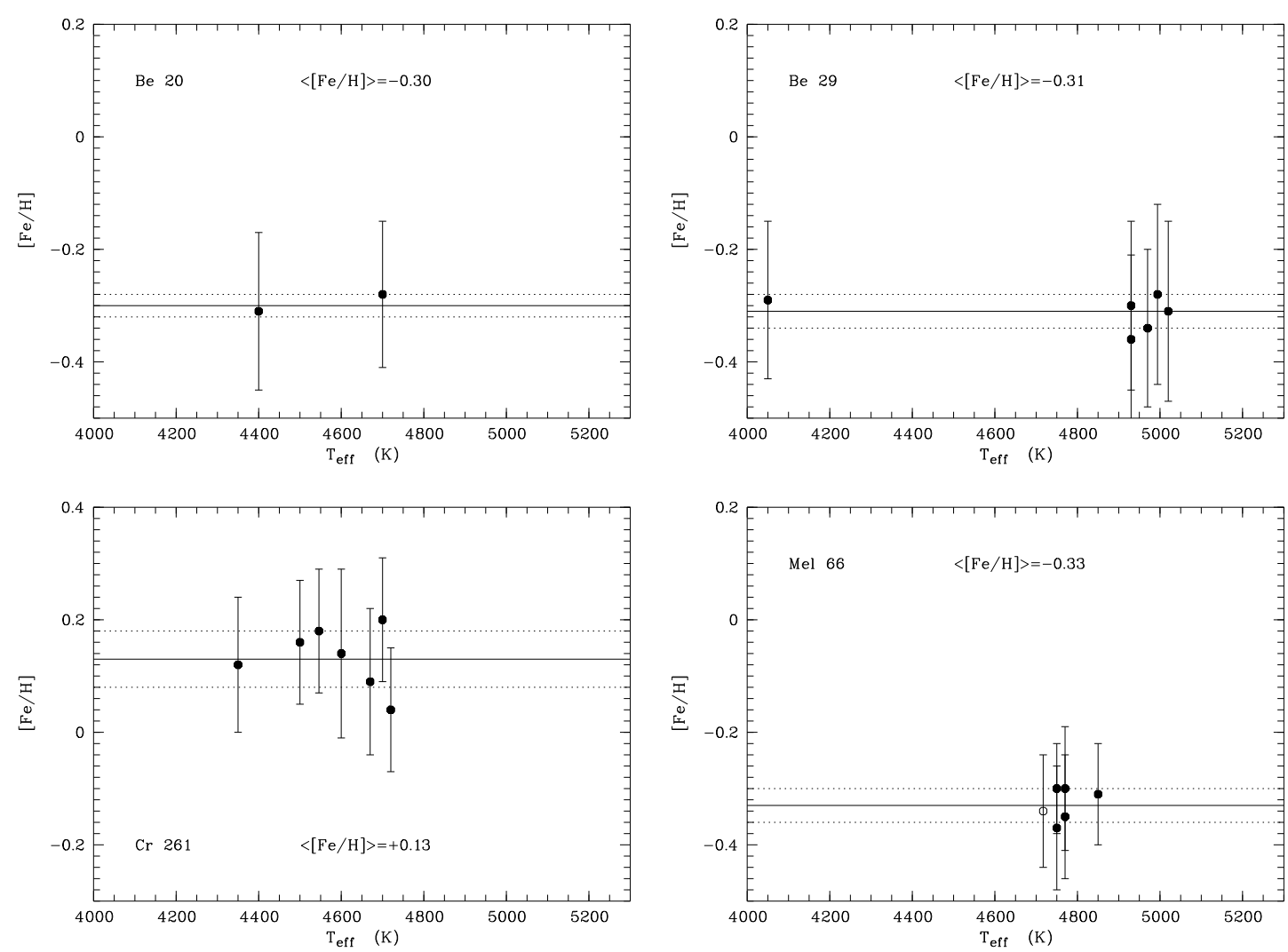

Fig. 3. Fe abundances as a function of $T_{\text {eff }}$ for members in the sample clusters. The solid line represents the average Fe content, while the dashed ones represent the rms. 
Table 9. $[\mathrm{X} / \mathrm{Fe}]$ abundances and averages. Errors are the quadratic sum of $\sigma_{1}$ on $[\mathrm{X} / \mathrm{H}]$ and on $[\mathrm{X} / \mathrm{Fe}]$.

\begin{tabular}{|c|c|c|c|c|c|c|}
\hline Star & {$[\mathrm{Si} \mathrm{I} / \mathrm{Fe}](\sigma)$} & {$[\mathrm{Ca} \mathrm{I} / \mathrm{Fe}](\sigma)$} & {$[\mathrm{Ti} \mathrm{I} / \mathrm{Fe}](\sigma)$} & {$[\mathrm{Cr} \mathrm{I} / \mathrm{Fe}](\sigma)$} & {$[\mathrm{Ni} \mathrm{I} / \mathrm{Fe}](\sigma)$} & {$[\mathrm{Ba} \mathrm{II} / \mathrm{Fe}](\sigma)$} \\
\hline \multicolumn{7}{|c|}{ Berkeley 20} \\
\hline 1201 & $+0.10(0.11)$ & $+0.12(0.17)$ & $+0.09(0.16)$ & $-0.10(0.20)$ & $+0.07(0.14)$ & $+0.07(0.14)$ \\
\hline 1240 & $-0.09(0.22)$ & $+0.03(0.16)$ & $+0.16(0.18)$ & $-0.10(0.17)$ & $-0.01(0.17)$ & $+0.26(0.14)$ \\
\hline \multirow[t]{2}{*}{ Average } & $+0.005(0.13)$ & $+0.075(0.06)$ & $+0.13(0.05)$ & $-0.10(0.00)$ & $+0.03(0.06)$ & $+0.16(0.13)$ \\
\hline & \multicolumn{6}{|c|}{ Berkeley 29} \\
\hline 159 & $-0.01(0.22)$ & $+0.09(0.18)$ & $-0.01(0.20)$ & $-0.10(0.18)$ & $-0.10(0.17)$ & $+0.40(0.25)$ \\
\hline 257 & $+0.13(0.28)$ & $+0.12(0.20)$ & $+0.02(0.20)$ & $-0.06(0.20)$ & $+0.01(0.18)$ & $+0.46(0.37)$ \\
\hline 398 & $-0.01(0.16)$ & $+0.14(0.18)$ & $+0.07(0.19)$ & $-0.10(0.20)$ & $-0.03(0.20)$ & $+0.46(0.40)$ \\
\hline 602 & $+0.05(0.12)$ & $+0.04(0.16)$ & $+0.06(0.15)$ & $-0.13(0.16)$ & $-0.08(0.13)$ & $+0.37(0.17)$ \\
\hline 933 & $-0.05(0.16)$ & $+0.08(0.19)$ & $-0.03(0.19)$ & $-0.11(0.18)$ & $-0.04(0.20)$ & $+0.37(0.20)$ \\
\hline 1024 & $+0.05(0.14)$ & $+0.12(0.15)$ & $+0.42(0.22)$ & $+0.03(0.29)$ & $+0.08(0.21)$ & $+0.53(0.12)$ \\
\hline \multirow[t]{2}{*}{ Average } & $+0.03(0.06)$ & $+0.10(0.04)$ & $+0.08(0.16)$ & $-0.08(0.06)$ & $-0.03(0.07)$ & $+0.43(0.06)$ \\
\hline & & & \multicolumn{4}{|c|}{$+0.02(0.04)$ : average [Ti/Fe] computed excluding star 1024} \\
\hline & & & Melotte 66 & & & \\
\hline 1346 & $+0.13(0.09)$ & $+0.15(0.18)$ & $-0.02(0.14)$ & -0.0 & $-0.02(0.11)$ & $+0.32(0.13)$ \\
\hline 1493 & $+0.14(0.10)$ & $+0.09(0.15)$ & $-0.02(0.14)$ & $+0.02(0.15)$ & $-0.01(0.14)$ & $+0.30(0.10)$ \\
\hline 1785 & $+0.10(0.10)$ & $+0.10(0.17)$ & $+0.01(0.13)$ & $-0.06(0.11)$ & $-0.05(0.12)$ & $+0.29(0.10)$ \\
\hline 1865 & $+0.14(0.11)$ & $+0.07(0.14)$ & $-0.05(0.12)$ & $-0.05(0.14)$ & $-0.02(0.11)$ & $+0.47(0.09)$ \\
\hline 1884 & $+0.10(0.10$ & $+0.06(0.13)$ & $+0.08(0.11)$ & $-0.02(0.13)$ & $0.00(0.09)$ & $+0.26(0.10)$ \\
\hline 2218 & $+0.16(0.11)$ & $+0.08(0.14)$ & $+0.01(0.11)$ & $+0.03(0.15)$ & $+0.03(0.11)$ & $+0.36(0.10)$ \\
\hline Average & $+0.13(0.02)$ & $+0.11(0.05)$ & $+0.02(0.04)$ & $-0.02(0.04)$ & $-0.01(0.03)$ & $+0.33(0.07)$ \\
\hline & & & Collinder 261 & & & \\
\hline RGB02 & $+0.17(0.22)$ & $+0.03(0.20)$ & $+0.24(0.20)$ & $+0.08(0.21)$ & $+0.08(0.18)$ & $+0.30(0.11)$ \\
\hline RGB05 & $+0.03(0.16)$ & $-0.04(0.20)$ & $-0.03(0.17)$ & $-0.05(0.18)$ & $0.00(0.16)$ & $+0.26(0.13)$ \\
\hline RGB06 & $+0.14(0.19)$ & $-0.12(0.14)$ & $+0.06(0.15)$ & $-0.04(0.17)$ & $+0.11(0.16)$ & $+0.29(0.12)$ \\
\hline RGB07 & $+0.03(0.25)$ & $-0.03(0.15)$ & $+0.22(0.19)$ & $+0.04(0.19)$ & $+0.10(0.17)$ & $+0.23(0.10)$ \\
\hline RGB09 & $+0.07(0.15)$ & $-0.03(0.16)$ & $+0.07(0.14)$ & $+0.10(0.18)$ & $+0.06(0.14)$ & $+0.31(0.09)$ \\
\hline RGB10 & $+0.09(0.12)$ & $-0.03(0.15)$ & $+0.14(0.16)$ & $+0.07(0.18)$ & $+0.04(0.17)$ & $+0.25(0.09)$ \\
\hline RGB11 & $+0.09(0.18)$ & $-0.07(0.16)$ & $-0.01(0.15)$ & $0.00(0.19)$ & $+0.11(0.17)$ & $+0.31(0.11)$ \\
\hline Average & $+0.09(0.05)$ & $-0.04(0.05)$ & $+0.10(0.10)$ & $+0.03(0.06)$ & $+0.07(0.04)$ & $+0.28(0.03)$ \\
\hline
\end{tabular}

agreement with other literature estimates and this test allowed us to conclude that no large systematic uncertainties affect our abundance scale.

\section{Results}

\subsection{Metallicity}

The results for the stellar parameters and Fe content in the three clusters are summarized in Table 8 , where we report the $T_{\text {eff }}$ and $\log g$ (both photometric - the starting values - and spectroscopic - the optimized values), the microturbulence $\xi$ (spectroscopic), $[\mathrm{Fe} / \mathrm{H}]$ and their errors $\left(\sigma_{1}\right.$ and the total $\sigma$, see Sect. 2.3), and the number of Fe I lines used for each star. There is generally good agreement between the photometric and spectroscopic temperatures. The largest discrepancies are for the two Be 20 stars for which the optimized $T_{\text {eff }}$ are $\sim 200 \mathrm{~K}$ larger than the initial ones, and for star 1493 in Mel 66 and star RGB05 in Cr 261, for which the spectroscopic $T_{\text {eff }}$ is about $150 \mathrm{~K}$ hotter than the photometric one. As for the surface gravities, for $\mathrm{Be} 20, \mathrm{Cr} 261$ and for three of the six stars in Mel 66 (1346, 1493 and 1785) the spectroscopic ones are lower than the initial $\log g$ with differences of $\sim 0.3$ dex (varying from 0.2 to 0.5 in the extreme cases). The discrepancy between spectroscopic and photometric $\log g$ was already discussed in Paper I, and can be ascribed to several random factors (internal errors, errors in distance moduli, in reddening values, in ages, etc.) or by non-LTE effects and/or inadequacies in classical (1-d) model atmospheres where important features such as spots, granulation, activity, etc. are neglected. Such a discrepancy has also been encountered by other authors (e.g., Feltzing \& Gustafsson 1998; Schuler et al. 2003; Allende Prieto et al. 2004) in studies of cool metal-rich stars. In the case of $\mathrm{Cr} 261$ the main sources of errors are the large uncertainties in the reddening (see also the discussion in Carretta et al. 2005). On the other hand, for stars in Be 29 we found a very good agreement among the two sets of gravities, with an average difference $-0.05 \pm 0.14$ dex. Estimates of the effects of variations in the stellar parameters on the derived abundances can be found in Table 13 of Paper I, and Table 12 of Paper II. The microturbulent velocities as optimized from the analysis are in very good agreement with the initial ones computed using the Carretta et al. (2004) formula for almost all the stars in the three sample, and for this reason we show only the final values in the table.

The average metallicities for the four clusters are listed in Table 8: three of them have subsolar Fe contents, that is $[\mathrm{Fe} / \mathrm{H}]=-0.30 \pm 0.02(\mathrm{Be} 20),-0.31 \pm 0.03(\mathrm{Be} 29),-0.33 \pm 0.03$ (Mel 66); Cr 261 has a slightly oversolar metallicity, similar to that of the Hyades $(+0.13 \pm 0.05)$. No significant scatter among the various stars in a single cluster exists. Note that star 1865 in Mel 66, labeled as a doubtful member for its $R V$ discrepant by more than three $\sigma$ from the average, has a metallicity in perfect agreement with those of other stars, therefore we consider this star as a possible member (perhaps a binary). Figure 3 shows plots of $[\mathrm{Fe} / \mathrm{H}]$ vs. effective temperature for the clusters. Error bars $\left(\sigma_{\text {tot }}\right)$ are reported, while the solid and dashed lines represent the average metallicity and the rms, respectively. We may appreciate the absence of any trend between temperature and metallicity; this is especially visible in the two clusters Be 29 and $\mathrm{Cr} 261$, where stars covering a larger range of temperatures were observed.

As briefly mentioned in Sect. 2.1, the previous (high resolution) spectroscopic metallicity determination for Be 20, Be 29 

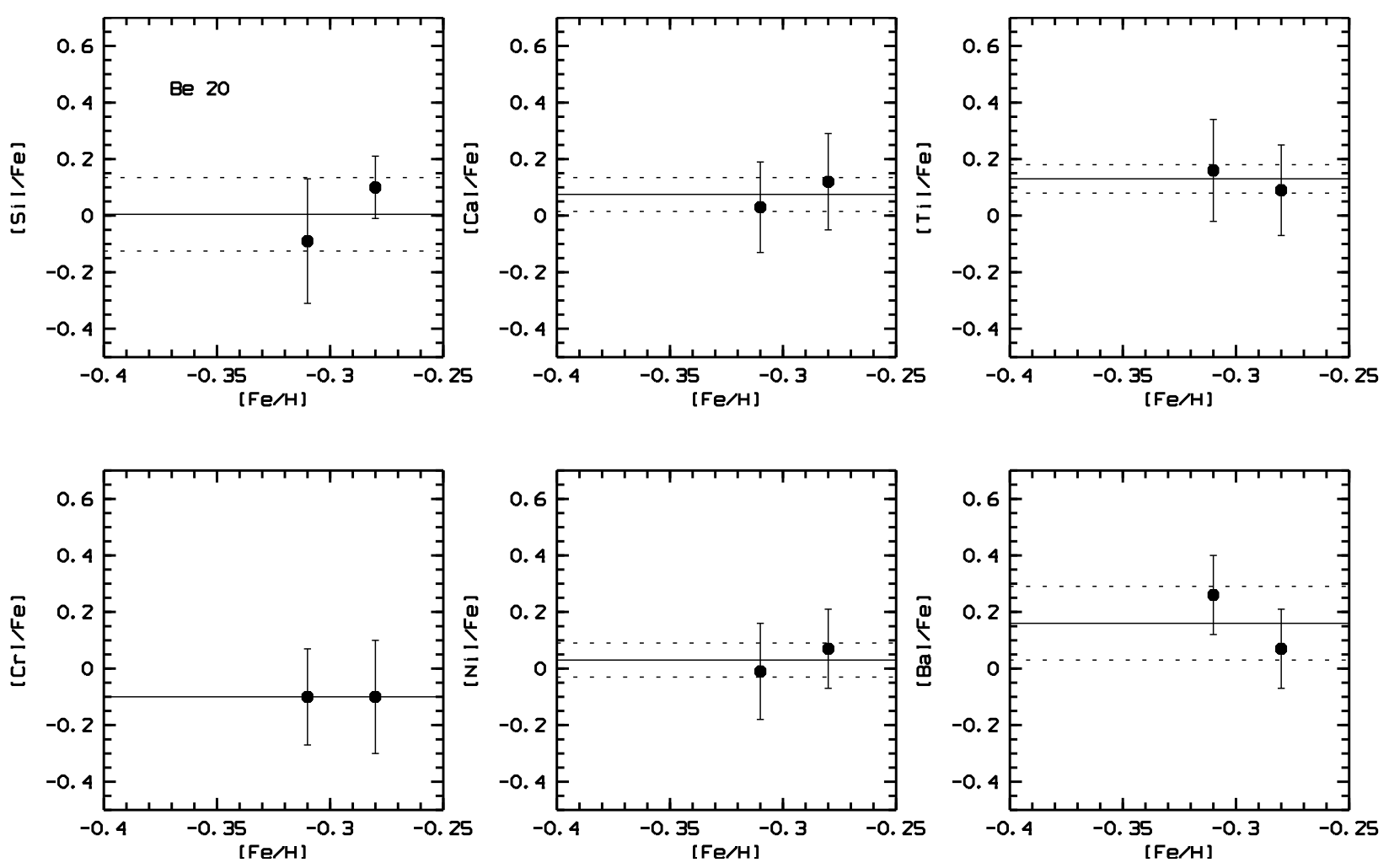

Fig. 4. $[\mathrm{X} / \mathrm{Fe}]$ abundance ratios as a function of $[\mathrm{Fe} / \mathrm{H}]$ for the two members in $\mathrm{Be} 20$.

and Mel 66 also found subsolar values, although somewhat lower than ours. In particular, Carraro et al. (2004) and Yong et al. (2005) determined $[\mathrm{Fe} / \mathrm{H}]=-0.44$ and -0.54 , respectively, for two stars each in Be 29. The value of $[\mathrm{Fe} / \mathrm{H}]=-0.38$ measured by Gratton \& Contarini (1994) for Mel 66 is instead closer to our value. For Cr 261 two previous analysis exist. Friel et al. (2003) obtained a metallicity $[\mathrm{Fe} / \mathrm{H}]=-0.22 \pm 0.05$, significantly lower than ours, while Carretta et al. (2005) found a solar [Fe/H].

\subsection{Abundances of other elements}

We derived the abundances of the light elements $\mathrm{Na}$ and $\mathrm{Al}$, the $\alpha$-elements $\mathrm{Mg}, \mathrm{Si}, \mathrm{Ca}$, and $\mathrm{Ti}$, the $\mathrm{Fe}$-peak elements $\mathrm{Cr}$ and $\mathrm{Ni}$, and the $s$-process element Ba. Table 9 shows the results $([\mathrm{X} / \mathrm{Fe}])$ for $\mathrm{Si}, \mathrm{Ca}, \mathrm{Ti}, \mathrm{Cr}, \mathrm{Ni}$, and $\mathrm{Ba}$, for which a significant number of lines was employed (see Paper II for further details).

The ratios of $\alpha$-elements $\mathrm{Si}, \mathrm{Ca}$, and Ti to $\mathrm{Fe}$ are close to solar in the sample clusters, with a slight enhancement of $\mathrm{Ca}$ and $\mathrm{Si}$ in some cases $([\mathrm{X} / \mathrm{Fe}] \sim+0.10 /+0.15)$. Also the $\mathrm{Fe}-$ peak elements are solar, with $[\mathrm{Cr} / \mathrm{Fe}]$ slightly below zero. The $s$-process $\mathrm{Ba}$ is sensitively enhanced, as already found by our group (Paper II; Bragaglia et al. 2006); abundance for this element, however, strongly depends on the adopted analysis and indeed has been found to vary a lot between clusters (e.g. Gratton et al. 2004). The $[\mathrm{X} / \mathrm{Fe}]$ values as a function of $[\mathrm{Fe} / \mathrm{H}]$ for $\mathrm{Si}$, $\mathrm{Ca}, \mathrm{Ti}, \mathrm{Cr}, \mathrm{Ni}$ and $\mathrm{Ba}$ are shown in Figs. 4-7 for the four clusters. No particular trends are present for abundances, nor a large scatter, with only a few exceptions: the two members of Be 20 have Si abundances differing by $\sim 0.2$ dex; the tip star in Be 29 (1024) has Ti abundance considerably higher than the average. The latter fact might be related to problems in determining abundances in such a cool star.

The determination of $\mathrm{Na}, \mathrm{Mg}$, and $\mathrm{Al}$ abundances is affected by larger uncertainties with respect to the other species above, since for these three elements only a few spectral lines are available in the wavelength range covered by our spectra, and moreover they are difficult to measure. Note that spectra in the $8000 \AA$ range were available only for $\mathrm{Cr} 261$ and Mel 66, as mentioned in Sect. 2. As already done in S07 and Paper II for Al and $\mathrm{Mg}$, we decided to present abundances on a line-by-line basis. The upper parts of Tables 10-13 show the abundances for each line of the elements $\mathrm{Al}, \mathrm{Mg}$, and $\mathrm{Na}$ (for the latter one, see next section). A large scatter is present among the different lines and the various stars, with some clear outliers being present. This is probably due to non-optimum continuum positioning related to quite low $S / N$.

\subsection{Sodium}

The abundances of $\mathrm{Na}, \mathrm{Mg}$, and $\mathrm{Al}$ computed by us using MOOG (LTE analysis) are in general oversolar (this is not an exception; for a recent determination see e.g., Jacobson et al. 2007). However, it is known that the Na spectral lines are affected by non-LTE effects, that depend on the temperature and evolutionary status of the star. For example, Gratton et al. (1999) computed non-LTE corrections (using statistical equilibrium calculations) for F- and K-type stars over a broad range of gravities. As shown in Paper II, the corrections following the prescription of Gratton et al. (1999) are about -0.02 to -0.10 dex for stars in our sample clusters. Slightly larger values are found following the prescription of the work by Mashonkina et al. (2000), who give corrections up to -0.15 dex for giant stars (see also S07).

A more recent analysis has been carried out by Andrievsky et al. (2007), who determined non-LTE Na abundances for a homogeneous sample of metal-poor stars with a direct method: namely, they compute non-LTE abundances by line profile fitting, and not by applying a correction to LTE calculated abundances. Andrievsky et al. (2007) use a modified version of the 
P. Sestito et al.: Abundances in old open clusters. III.
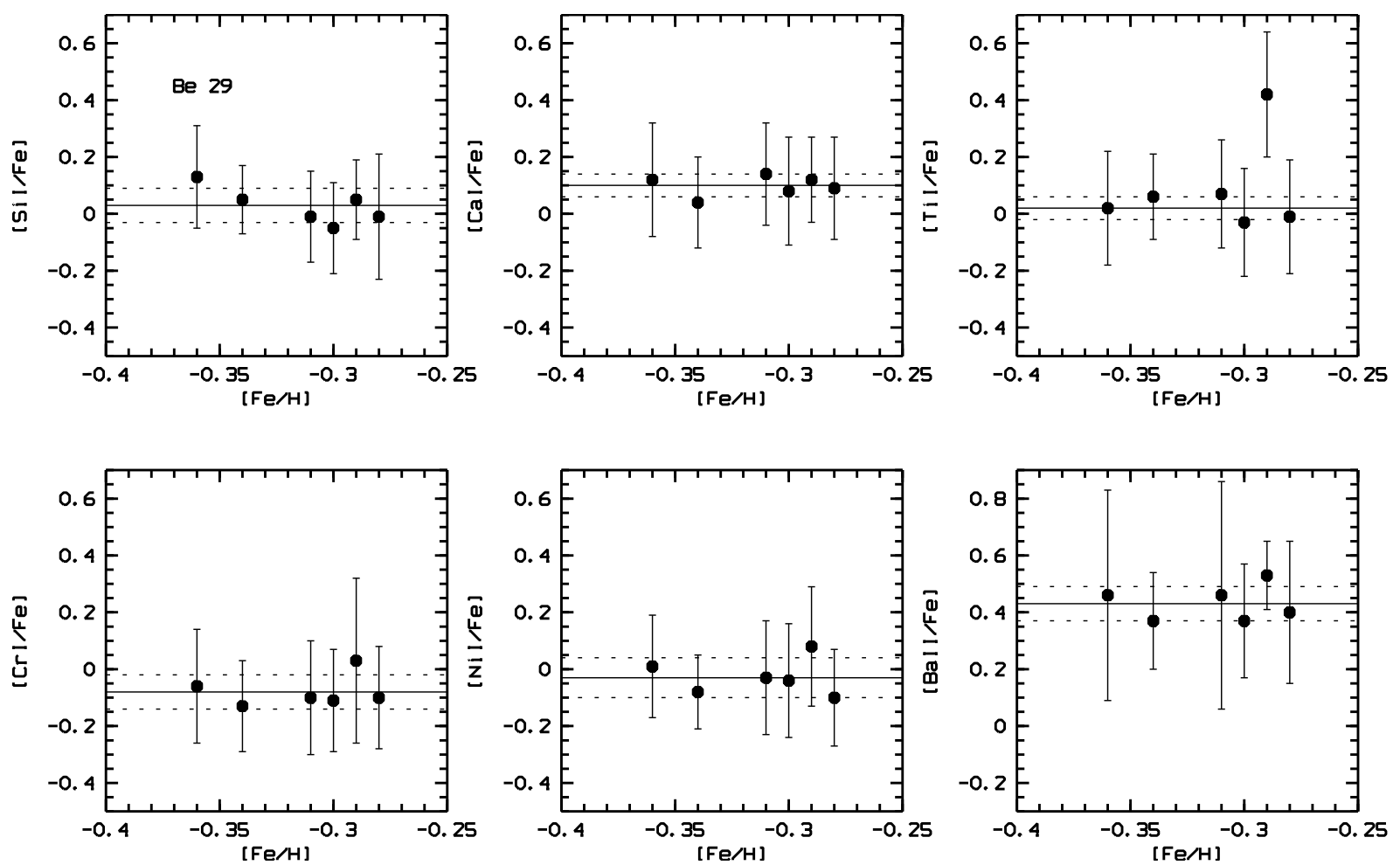

Fig. 5. $[\mathrm{X} / \mathrm{Fe}]$ vs. $[\mathrm{Fe} / \mathrm{H}]$ for stars in Be 29. The average $[\mathrm{Ti} / \mathrm{Fe}]$ has been computed excluding the tip star 1024 , since we derived a very high abundance for this element.
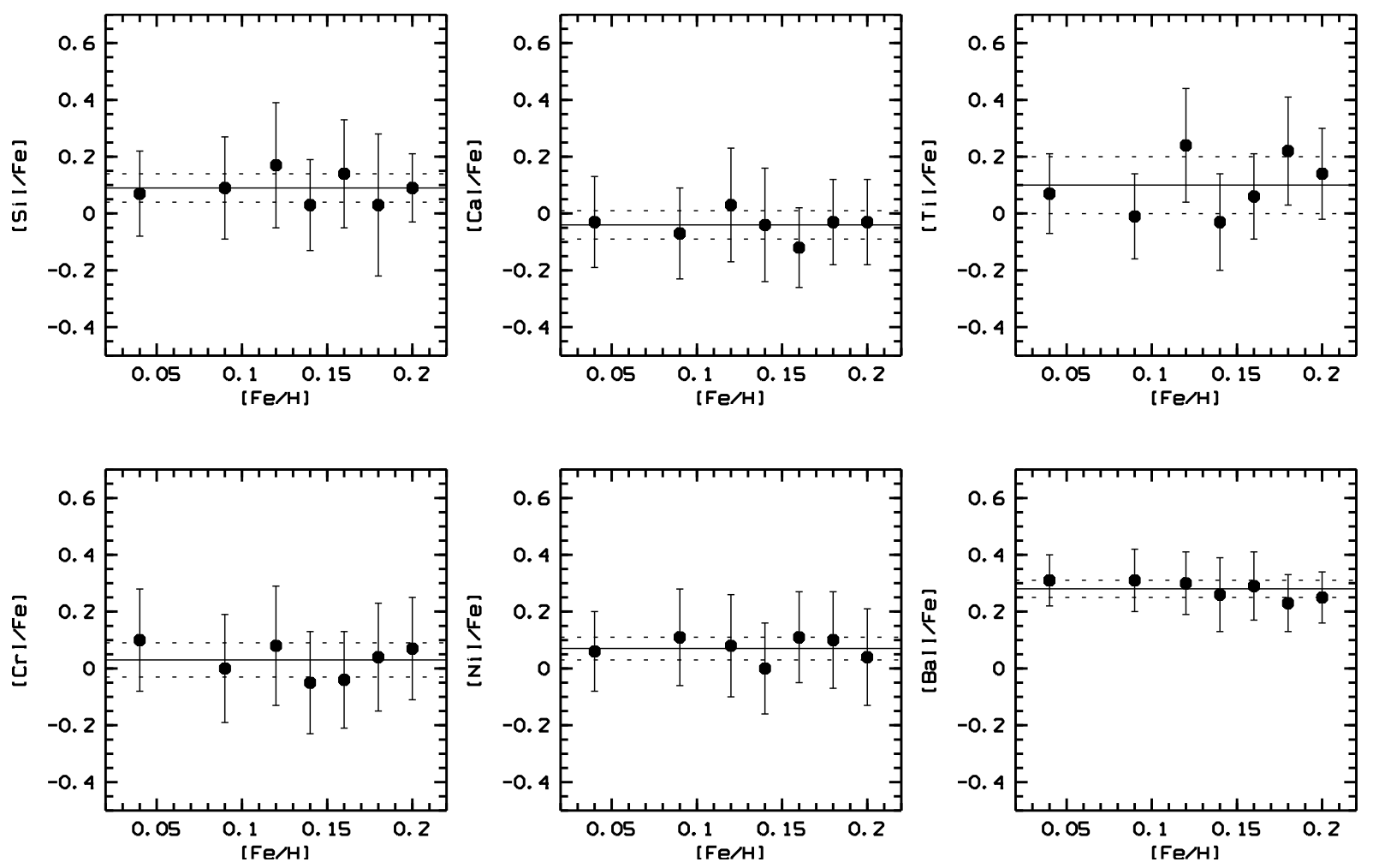

Fig. 6. $[\mathrm{X} / \mathrm{Fe}]$ vs. $[\mathrm{Fe} / \mathrm{H}]$ for stars in $\mathrm{Cr} 261$.

code MULTI (Carlsson et al. 1986; Korotin et al. 1999a; Korotin et al. 1999b; see additional details in Andrievsky et al. 2007). The atomic model that consists of 27 energy levels of $\mathrm{Na}$ I atom and the ground level of Na II ion was used in our NLTE calculations. The radiative transitions between the first 20 levels of $\mathrm{Na}$ I and the ground level of Na II are considered. Transitions between the other levels are used only for the particle number conservation. Linearizing procedure includes $46 b-b$ and $20 b-f$ transitions. Radiative rates for 34 transitions are fixed.

As mentioned in Sect. 3.2, in the upper parts of Tables 10-13 we show the $\mathrm{Na}$ abundances for each line computed using MOOG (LTE). In the last lines of the same tables, we report the 

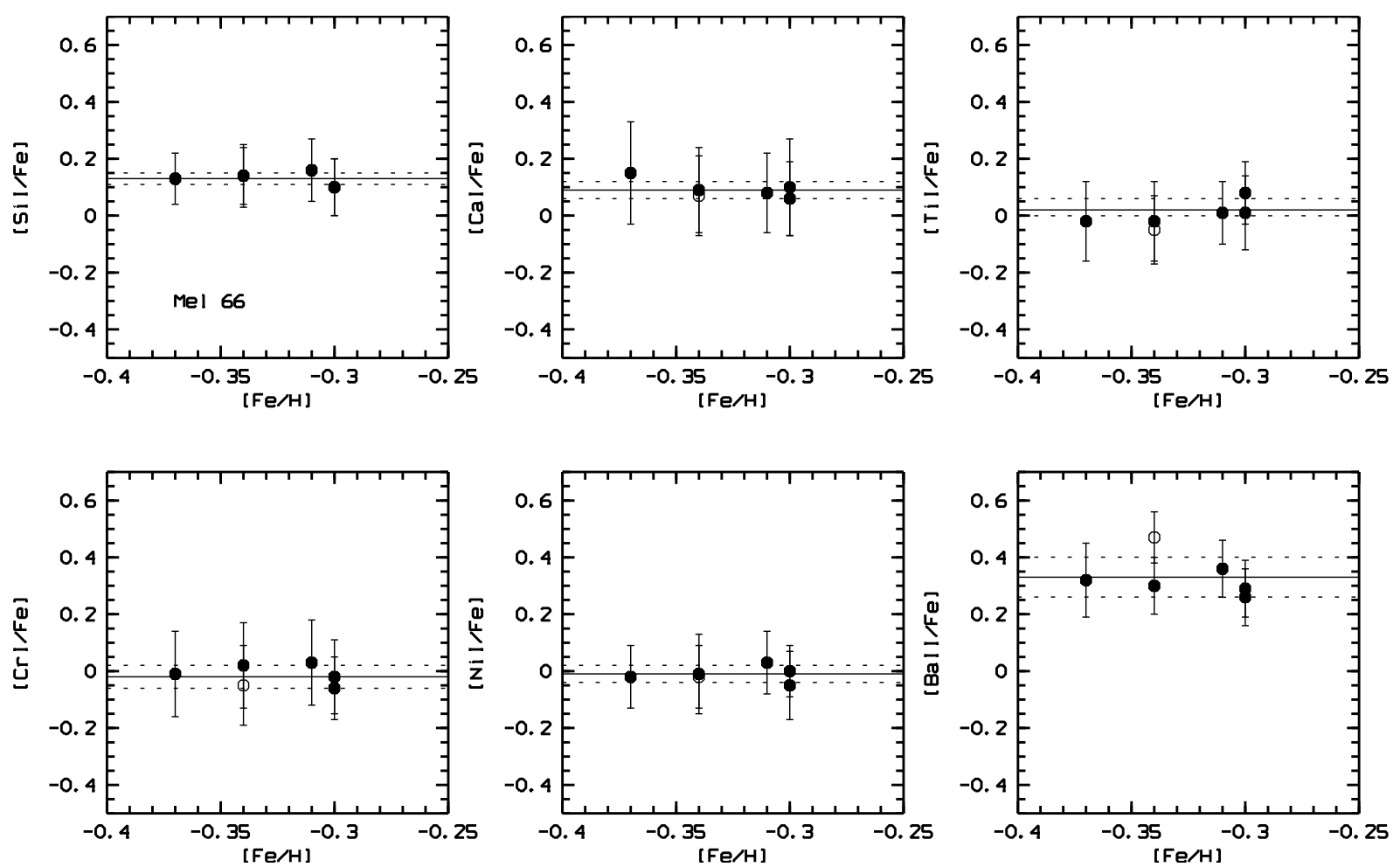

Fig. 7. $[\mathrm{X} / \mathrm{Fe}]$ vs. $[\mathrm{Fe} / \mathrm{H}]$ for stars in $\mathrm{Mel} 66$. The open symbol represents the probable member.

Table 10. Abundances of Na (LTE and non-LTE), Mg, and Al for stars in Be 20. The mean non-LTE Na abundances shown in the last line were computed from line profile fitting.

\begin{tabular}{|c|c|c|c|c|c|}
\hline \multicolumn{6}{|l|}{$\overline{\overline{B e} 20}$} \\
\hline Wavelength & $\log n(\mathrm{X})_{\odot}$ & {$[\mathrm{X} / \mathrm{H}]$} & {$[\mathrm{X} / \mathrm{Fe}]$} & {$[\mathrm{X} / \mathrm{H}]$} & {$[\mathrm{X} / \mathrm{Fe}]$} \\
\hline \multicolumn{6}{|c|}{$M g_{I}$} \\
\hline 6318.71 & 7.54 & 7.51 & +0.25 & 7.62 & +0.39 \\
\hline 6319.24 & 7.47 & - & - & 7.36 & +0.20 \\
\hline \multicolumn{6}{|c|}{$\mathrm{Al} \mathrm{I}$} \\
\hline 6696.032 & 6.29 & 6.14 & +0.13 & 6.31 & +0.33 \\
\hline 6698.669 & 6.18 & 5.93 & +0.03 & 6.04 & +0.17 \\
\hline \multicolumn{6}{|c|}{ Na I(LTE) } \\
\hline 5688.22 & 6.20 & 6.25 & +0.33 & 5.71 & -0.18 \\
\hline 6154.23 & 6.29 & 6.34 & +0.33 & 6.20 & +0.22 \\
\hline 6160.75 & 6.30 & 6.14 & +0.12 & 6.18 & +0.19 \\
\hline & & {$[\mathrm{Na} / \mathrm{H}]_{\mathrm{NLTE}}$} & {$[\mathrm{Na} / \mathrm{Fe}]_{\text {NLTE }}$} & {$[\mathrm{Na} / \mathrm{H}]_{\text {NLTE }}$} & {$[\mathrm{Na} / \mathrm{Fe}]_{\text {NLTE }}$} \\
\hline Average $\mathrm{Na}$ & & $5.90 \pm 0.10$ & $-0.07 \pm 0.14$ & $5.90 \pm 0.10$ & $-0.04 \pm 0.16$ \\
\hline
\end{tabular}

non-LTE average abundances, computed by fitting the profiles of the same $\mathrm{Na}$ lines used in the $E W$ analysis. The spectroscopic stellar parameters derived by us were adopted, which provide good fits to the lines. We show both the $[\mathrm{Na} / \mathrm{H}]_{\text {non-LTE }}$ values derived from line fitting and the $[\mathrm{Na} / \mathrm{Fe}]_{\text {non-LTE }}$ abundances: these ones were computed by using $[\mathrm{Fe} / \mathrm{H}]$ derived by us for each star, and $\log n(\mathrm{Na})_{\odot}=6.25$ obtained by Andrievsky et al. (2007) with the same line fitting method (non-LTE). As clearly visible, the final $\mathrm{Na}$ abundances including non-LTE effects are nearly solar (or even lower), i.e. no $\mathrm{Na}$ enhancement is seen.

Figure 8 shows $[\mathrm{Na} / \mathrm{Fe}]_{\text {non-LTE }} \mathrm{vs}$. $[\mathrm{Fe} / \mathrm{H}]$ for the open clusters analyzed so far by our group: the clusters of Papers I and II (NGC 3960, NGC 2660, NGC 2324, NGC 2477 and Be 32) for which we adopted the corrections by Gratton et al. (1999); NGC 6253 (S07) for which we used the corrections by Mashonkina et al. (2000), and the clusters presented in this paper, whose non-LTE abundances were calculated with the method of Andrievsky et al. (2007). We can immediately appreciate the difference between the different non-LTE corrections, since all the stars in the various clusters have similar temperature and evolutionary status: the new computations produce $[\mathrm{Na} / \mathrm{Fe}]$ values lower by about $0.1-0.2$ dex. However, our previous measurements were only slightly oversolar, with an average $[\mathrm{Na} / \mathrm{Fe}]$ of about +0.1 dex.

In most studies of abundances in giant stars a Na enhancement was found (e.g., Friel et al. 2003; Yong et al. 2005; Carretta et al. 2005; Jacobson et al. 2007) and some discuss the possibility that it is a real feature (e.g. Pasquini et al. 2004). However, our computations seem to favor the hypothesis that oversolar $[\mathrm{Na} / \mathrm{Fe}]$ might rather be a consequence of a non-adequate treatment of non-LTE effects for giant stars. The validity of this hypothesis is reinforced by the fact that the enhancement is not seen in unevolved cluster stars and in field dwarfs; see e.g. Randich et al. (2006) for M 67, where they also conclude that dwarfs and 

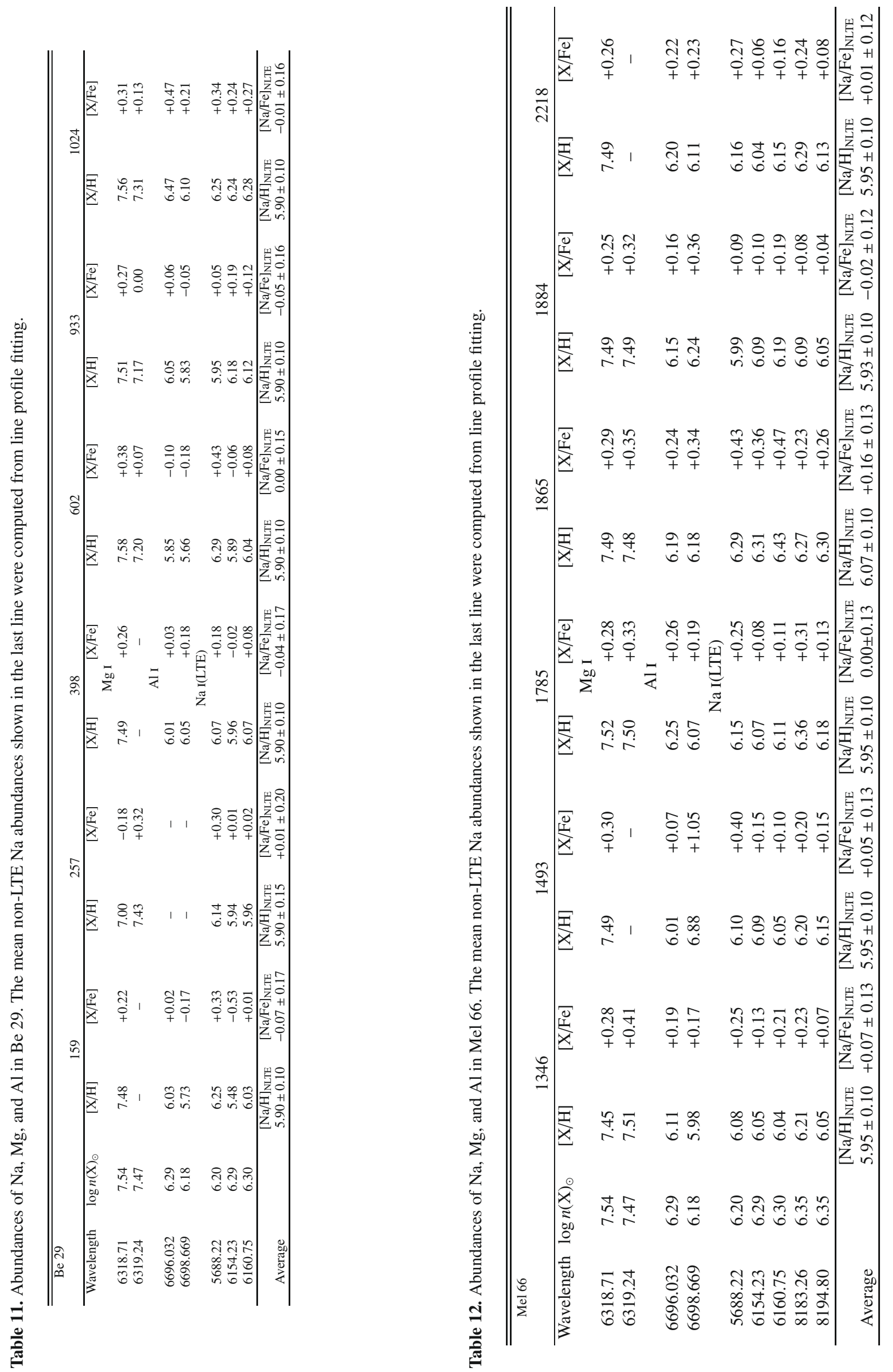


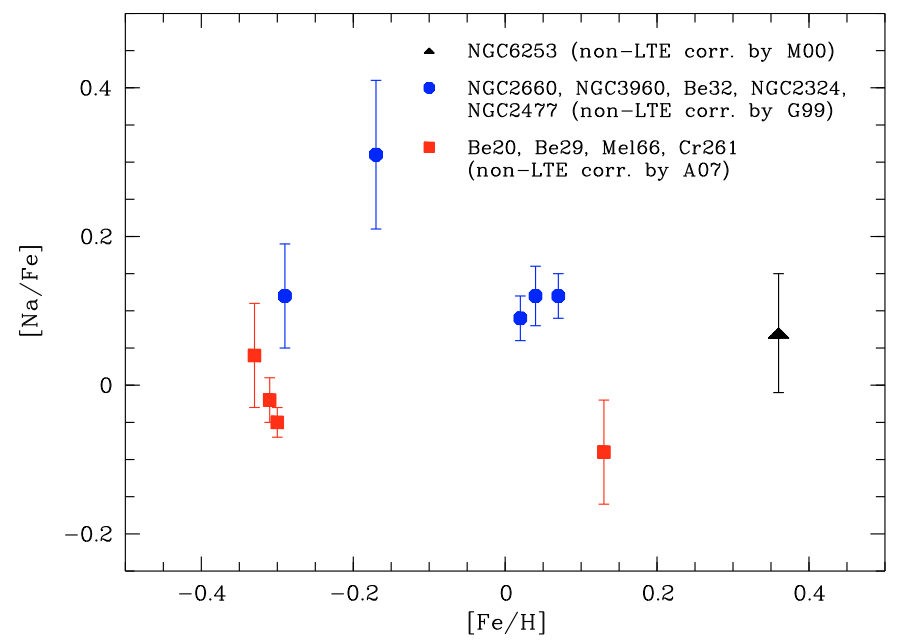

Fig. 8. $[\mathrm{Na} / \mathrm{Fe}]_{\text {non-LTE }}$ vs. $[\mathrm{Fe} / \mathrm{H}]$ for clusters analyzed by us: circles are clusters of Papers I and II, for which we adopted the corrections by Gratton et al. (1999); the triangle is NGC 6253 (S07) for which we used the corrections by Mashonkina et al. (2000), and squares are clusters presented in this paper, whose abundances were computed following the profile fitting method by Andrievsky et al. (2007).

giants have the same abundances, and Soubiran \& Girard (2005) for field stars. However, recently, Mishenina et al. (2006) studied about 180 field red clump stars and measured non-LTE Na abundances in the same way as in the present paper finding a quite large dispersion (see their Fig. 14) and an average overabundance of about +0.1 dex; they also note that the $[\mathrm{Na} / \mathrm{Fe}]$ abundances do not behave in the same way for field dwarfs and giants. The problem is not simple, and a dedicated study would be welcome.

\section{Discussion}

In Table 14 we give a summary of the properties and abundances of some key elements in the open clusters analyzed by our group (this paper, Papers I, II and S07). The Galactocentric radii are from Friel et al. (2002), when present; otherwise they were taken from Friel (1995), Friel (2006). Ages are from Bragaglia \& Tosi (2006), Bragaglia et al. (2006), Tosi et al. (2007). The left panel of Fig. 9 shows the distribution of $[\mathrm{Fe} / \mathrm{H}]$ as a function of the Galactocentric distance: the sample includes our data and other clusters analyzed with high resolution spectroscopy ${ }^{4}$. The right panel of Fig. 9 shows the radial $[\mathrm{Fe} / \mathrm{H}]$ distribution for open clusters observed at low resolution by Friel et al. (2002). The $[\mathrm{Fe} / \mathrm{H}]$

4 Literature high resolution studies: Smith \& Suntzeff (1987: NGC 2420), Gratton \& Contarini (1994: NGC 2243), Brown et al. (1996: Mel 71, To 2), Hamdani et al. (2000: NGC 2360, NGC 2447), Gonzalez \& Wallerstein (2000: M 11), Bragaglia et al. (2001: NGC 6819), Randich et al. (2001: IC 2602, IC 2391; 2003: NGC 188; 2006: M 67), Sestito et al. (2003: NGC 6475), Paulson et al. (2003: Hyades), Schuler et al. (2003: M 34; 2004: Pleiades), Carretta et al. (2004: IC 4651, NGC 2506, NGC 6134), Carraro et al. (2004: Saurer 1; 2007: Be 25, Be 73, Be 75, Rup 4, Rup 7), Ford et al. (2005: Blanco 1), Tautvaisiene et al. (2005: NGC 7789), Villanova et al. (2005: Be 22, Be 66), Yong et al. (2005: Be 31, NGC 2141), Pace et al. (in prep.: Praesepe). The point representative of NGC 6791 (the most metal rich cluster) is an average between the $[\mathrm{Fe} / \mathrm{H}]$ values obtained by Carraro et al. (2006b), Gratton et al. (2006), and Origlia et al. (2006). We included in the plot only clusters older than $\sim 100 \mathrm{Myr}$; in the cases of clusters in common between us and other authors, we chose our own metallicity determination. Most $[\mathrm{Fe} / \mathrm{H}]$ values are based on the analysis of giant stars, except those for the young values in the two panels of Fig. 9 are taken directly from the literature sources: thus, whereas the low resolution data by Friel et al. represents a homogeneous sample, our data and the other high resolution ones from the literature could be on different scales. Whereas this might introduce some spurious scatter, some general features are evident in the figure. If one considers high resolution data, there appears to be a quite steep metallicity gradient up to a Galactocentric distance $R_{\mathrm{gc}} \lesssim 10-11 \mathrm{kpc}$, and then a flattening at larger distances. More in detail, using a weighted linear fit, we find a slope of $-0.17 \pm 0.02 \mathrm{dex} \mathrm{kpc}^{-1}$ (with a correlation coefficient -0.83 ) in the disk region with $R_{\mathrm{gc}} \leq 11 \mathrm{kpc}$, while in the outer disk $\left(R_{\mathrm{gc}}>11 \mathrm{kpc}\right)$ the slope is, within the errors, consistent with zero. The sample by Friel et al. (2002) is instead limited to $R_{\mathrm{gc}} \lesssim 16 \mathrm{kpc}$, with only one cluster in the distance range $R_{\mathrm{gc}} \sim 14-16 \mathrm{kpc}$ : these authors found a negative slope of $-0.063 \pm 0.010 \mathrm{dex} \mathrm{kpc} \mathrm{kp}^{-1}$ between $\sim 7$ and $16 \mathrm{kpc}$, and they did not discuss the possibility of a flattening of the gradient outside of a given radius. Considering only the clusters at $R_{\mathrm{gc}} \leq 11 \mathrm{kpc}$, the $[\mathrm{Fe} / \mathrm{H}]$ gradient from low resolution data has a slope of $-0.09 \pm 0.02 \mathrm{dex} \mathrm{kpc}^{-1}$. The difference between this value of the slope in the inner disk and that found from high resolution data within the same radius $\left(R_{\mathrm{gc}} \leq 11 \mathrm{kpc}\right)$ is important, since even small differences in the slope might lead to changes in the infall rate and star formation efficiency adopted in the models. This point will be discussed in more detail in a future paper of the series, where we plan to compare our empirical results with updated Galactic chemical evolution models. On the other hand, considering only high resolution data, our sample and the one from the literature give similar results; in particular, the possibility of a plateau in the Fe gradient for clusters in the outer disk was already evidenced by Yong et al. (2005) who studied Be 20 and Be 29 (also in our sample), and two clusters at $R_{\mathrm{gc}} \sim 12 \mathrm{kpc}, \mathrm{Be} 31$ and NGC 2141, as well as by Carraro et al. $(2004,2007)$. Our clusters significantly improve the statistics, confirming on a more solid basis the change in the slope of the gradient, and in particular of a flattening for radii larger than $\sim 11 \mathrm{kpc}$; the average metallicity outside of this Galactocentric distance is $-0.27 \pm 0.13$. However, we have to remember that the "inner" slope is based on a short baseline (about $4 \mathrm{kpc}$ ), since the OC closest to the Galactic centre has $R_{\mathrm{GC}}$ slightly less than $7 \mathrm{kpc}$, and that the number or "outer" OCs is still scarce. To improve the situation, we should concentrate on deriving the metallicity of clusters both nearer to the Galactic centre and in the outer part of the disk, and in the transition region between the two slopes. The growing number of photometric studies dedicated to overlooked OCs (e.g., Carraro et al. 2006a, 2007; Ortolani et al. 2005) and the new catalogues of candidate clusters (e.g., Froebrich et al. 2007; Kronberger et al. 2006) will provide a good database for future abundance studies.

Figure 10 shows $[\mathrm{X} / \mathrm{Fe}]$ ratios vs. $[\mathrm{Fe} / \mathrm{H}]$ for the open clusters in our sample and for field disk stars. We show the $\alpha$-elements $\mathrm{Si}, \mathrm{Ca}, \mathrm{Ti}$ and the Fe-peak elements $\mathrm{Cr}$ and $\mathrm{Ni}$. We considered the disk star catalogue by Soubiran \& Girard (2005), which is a compilation and rehomogenization of several recent literature studies, but we also included the data by Bensby et al. (2005), since the latter authors derived also $\mathrm{Cr}$ abundances, not included in the other catalogue. It is evident that the open clusters follow the same distribution of chemical abundances with $[\mathrm{Fe} / \mathrm{H}]$ of disk stars, with average values close to (but slightly larger than) solar. Note that the $\alpha$-elements have a certain amount of scatter, in particular Ti.

clusters IC 2602, IC 2391, Pleiades, Blanco 1, NGC 6475, M34, Hyades, and for the old clusters M67 and NGC 188. 
Table 13. Abundances of $\mathrm{Na}, \mathrm{Mg}$, and $\mathrm{Al}$ in $\mathrm{Cr} 261$. The mean non-LTE $\mathrm{Na}$ abundances shown in the last line were computed from line profile fitting.

\begin{tabular}{|c|c|c|c|c|c|c|c|c|c|}
\hline \multicolumn{10}{|l|}{ Cr 261} \\
\hline & & \multicolumn{2}{|c|}{02} & \multicolumn{2}{|c|}{05} & \multicolumn{2}{|c|}{06} & \multicolumn{2}{|c|}{07} \\
\hline Wavelength & $\log n(\mathrm{X})_{\odot}$ & {$[\mathrm{X} / \mathrm{H}]$} & {$[\mathrm{X} / \mathrm{Fe}]$} & {$[\mathrm{X} / \mathrm{H}]$} & {$[\mathrm{X} / \mathrm{Fe}]$} & {$[\mathrm{X} / \mathrm{H}]$} & {$[\mathrm{X} / \mathrm{Fe}]$} & {$[\mathrm{X} / \mathrm{H}]$} & {$[\mathrm{X} / \mathrm{Fe}]$} \\
\hline \multicolumn{10}{|c|}{ Mg I } \\
\hline 6318.71 & 7.54 & 7.81 & +0.15 & 7.90 & +0.22 & 7.84 & +0.14 & 7.94 & +0.22 \\
\hline 6319.24 & 7.47 & 7.90 & +0.31 & 7.98 & +0.37 & 7.92 & +0.29 & 7.26 & -0.39 \\
\hline \multicolumn{10}{|c|}{$\mathrm{Al} \mathrm{I}$} \\
\hline 6696.032 & 6.29 & 6.81 & +0.40 & 6.60 & +0.17 & 6.64 & +0.19 & 6.80 & +0.33 \\
\hline 6698.669 & 6.18 & 5.52 & +0.22 & 6.37 & +0.05 & 6.74 & +0.40 & 6.68 & +0.32 \\
\hline \multicolumn{10}{|c|}{ Na I(LTE) } \\
\hline 5688.22 & 6.20 & 6.45 & +0.13 & 6.31 & -0.03 & 6.31 & -0.05 & 6.43 & +0.05 \\
\hline 6154.23 & 6.29 & 6.64 & +0.23 & 6.38 & -0.01 & 6.56 & +0.11 & 6.49 & +0.02 \\
\hline 6160.75 & 6.30 & 6.58 & +0.16 & 6.43 & +0.01 & 6.52 & +0.06 & 6.64 & +0.16 \\
\hline 8183.26 & 6.35 & 6.57 & +0.20 & 6.50 & +0.01 & 6.38 & -0.13 & 6.46 & -0.07 \\
\hline 8194.80 & 6.35 & 6.60 & +0.23 & 6.50 & +0.02 & 6.43 & -0.08 & 6.57 & +0.04 \\
\hline Average & & $\begin{array}{c}{[\mathrm{Na} / \mathrm{H}]_{\mathrm{NLTE}}} \\
6.35 \pm 0.07\end{array}$ & $\begin{array}{c}{[\mathrm{Na} / \mathrm{Fe}]_{\mathrm{NLTE}}} \\
-0.02 \pm 0.12\end{array}$ & $\begin{array}{c}\mathrm{Na} / \mathrm{H}]_{\mathrm{NLTE}} \\
6.20 \pm 0.07\end{array}$ & $\begin{array}{c}\mathrm{Na} / \mathrm{Fe}]_{\text {NLTE }} \\
-0.19 \pm 0.15\end{array}$ & $\begin{array}{c}\mathrm{Na} / \mathrm{H}]_{\mathrm{NLTE}} \\
6.30 \pm 0.07\end{array}$ & $\begin{array}{c}\mathrm{Na} / \mathrm{Fe}]_{\mathrm{NLTE}} \\
-0.11 \pm 0.11\end{array}$ & $\begin{array}{c}\mathrm{Na} / \mathrm{H}]_{\mathrm{NLTE}} \\
6.38 \pm 0.07\end{array}$ & $\begin{array}{c}\mathrm{Na} / \mathrm{Fe}]_{\text {NLTE }} \\
-0.05 \pm 0.11\end{array}$ \\
\hline
\end{tabular}

\begin{tabular}{|c|c|c|c|c|c|c|c|}
\hline \multicolumn{8}{|l|}{ Cr 261} \\
\hline & & \multicolumn{2}{|c|}{09} & \multicolumn{2}{|c|}{10} & \multicolumn{2}{|c|}{11} \\
\hline Wavelength & $\log n(\mathrm{X})_{\odot}$ & {$[\mathrm{X} / \mathrm{H}]$} & {$[\mathrm{X} / \mathrm{Fe}]$} & {$[\mathrm{X} / \mathrm{H}]$} & {$[\mathrm{X} / \mathrm{Fe}]$} & {$[\mathrm{X} / \mathrm{H}]$} & {$[\mathrm{X} / \mathrm{Fe}]$} \\
\hline \multicolumn{8}{|c|}{ Mg I } \\
\hline 6318.71 & 7.54 & 7.73 & +0.15 & 7.91 & +0.17 & 7.89 & +0.26 \\
\hline 6319.24 & 7.47 & 7.79 & +0.28 & 7.95 & +0.28 & 7.94 & +0.38 \\
\hline \multicolumn{8}{|c|}{$\mathrm{Al}$ I } \\
\hline 6696.032 & 6.29 & 6.62 & +0.29 & 6.84 & +0.35 & 6.69 & +0.31 \\
\hline 6698.669 & 6.18 & 6.36 & +0.14 & 6.58 & +0.20 & 6.53 & +0.26 \\
\hline \multicolumn{8}{|c|}{$\mathrm{Na}$ I(LTE) } \\
\hline 5688.22 & 6.20 & 6.42 & +0.18 & 6.49 & +0.09 & 6.28 & 0.00 \\
\hline 6154.23 & 6.29 & 6.41 & +0.07 & 6.68 & +0.19 & 6.39 & +0.08 \\
\hline 6160.75 & 6.30 & 6.46 & +0.12 & 6.57 & +0.07 & 6.47 & -0.03 \\
\hline 8183.26 & 6.35 & 6.47 & +0.08 & 6.50 & -0.04 & 6.41 & 0.00 \\
\hline 8194.80 & 6.35 & 6.45 & +0.05 & 6.47 & -0.08 & 6.44 & +0.26 \\
\hline \multirow{2}{*}{\multicolumn{2}{|c|}{ Average }} & {$[\mathrm{Na} / \mathrm{H}]_{\mathrm{NLTE}}$} & {$[\mathrm{Na} / \mathrm{Fe}]_{\mathrm{NLTE}}$} & {$[\mathrm{Na} / \mathrm{H}]_{\mathrm{NLTE}}$} & {$[\mathrm{Na} / \mathrm{Fe}]_{\mathrm{NLTE}}$} & {$[\mathrm{Na} / \mathrm{H}]_{\mathrm{NLTE}}$} & {$[\mathrm{Na} / \mathrm{Fe}]_{N L T E}$} \\
\hline & & $6.27 \pm 0.07$ & $-0.02 \pm 0.11$ & $6.32 \pm 0.07$ & $-0.13 \pm 0.11$ & $6.20 \pm 0.07$ & $-0.14 \pm 0.13$ \\
\hline
\end{tabular}

Table 14. The open clusters in our sample and their main properties.

\begin{tabular}{|c|c|c|c|c|c|c|c|c|c|c|}
\hline$\overline{\text { Cluster }}$ & $\begin{array}{l}\text { Age } \\
(\mathrm{Gyr})\end{array}$ & $\begin{array}{r}R_{\mathrm{gc}} \\
(\mathrm{kpc})\end{array}$ & $\overline{[\mathrm{Fe} / \mathrm{H}]}$ & $\overline{[\mathrm{Si} / \mathrm{Fe}]}$ & $\overline{[\mathrm{Ca} / \mathrm{Fe}]}$ & $\overline{[\mathrm{Ti} / \mathrm{Fe}]}$ & $\overline{[\mathrm{Cr} / \mathrm{Fe}]}$ & $\overline{[\mathrm{Ni} / \mathrm{Fe}]}$ & $\overline{[\mathrm{Ba} / \mathrm{Fe}]}$ & $\overline{\text { Ref. }}$ \\
\hline NGC 6253 & 3.0 & 6.60 & $+0.36 \pm 0.07$ & $01 \pm 0.05$ & .04 & 0.15 & $\overline{.04} \pm 0.05$ & $0.05 \pm 0.05$ & $.23 \pm 0.10$ & S07 \\
\hline Cr 261 & 6.0 & 7.52 & $+0.13 \pm 0.05$ & $+0.09 \pm 0.05$ & $-0.04 \pm 0.05$ & $10 \pm 0.10$ & $+0.03 \pm 0.06$ & $+0.07 \pm 0.04$ & $+0.28 \pm 0.03$ & This \\
\hline NGC 3960 & 0.9 & 7.96 & $+0.02 \pm 0.04$ & $+0.04 \pm 0.05$ & $+0.02 \pm 0.03$ & $-0.04 \pm 0.02$ & $+0.05 \pm 0.03$ & $-0.01 \pm 0.03$ & $+0.54 \pm 0.04$ & Paper I \\
\hline NGC 2477 & 1.0 & 8.94 & $+0.07 \pm 0.03$ & $+0.05 \pm 0.03$ & $-0.01 \pm 0.01$ & $+0.01 \pm 0.06$ & $+0.02 \pm 0.03$ & $0.00 \pm 0.04$ & $+0.46 \pm 0.05$ & Paper II \\
\hline NGC 2660 & 1.0 & 9.18 & $+0.04 \pm 0.04$ & $0.00 \pm 0.03$ & $+0.04 \pm 0.05$ & 0.03 & $+0.00 \pm 0.02$ & $-0.03 \pm 0.02$ & $+0.61 \pm 0.04$ & Paper I \\
\hline Mel 66 & 4.0 & 10.21 & \pm 0.03 & $+0.13 \pm 0.02$ & .05 & 04 & $2 \pm 0.04$ & $-0.01 \pm 0.03$ & $+0.33 \pm 0.07$ & This paper \\
\hline Be 32 & 5.5 & 11.35 & $-0.29 \pm 0.04$ & $+0.12 \pm 0.04$ & .04 & \pm 0.06 & $5 \pm 0.04$ & $0.00 \pm 0.04$ & $+0.29 \pm 0.10$ & Paper I \\
\hline NGC 2324 & 0.6 & 11.65 & $-0.17 \pm 0.05$ & $+0.06 \pm 0.11$ & 05 & -0 & 0.03 & $-0.09 \pm 0.02$ & $+0.62 \pm 0.08$ & Paper II \\
\hline Be 20 & 6.0 & 16.37 & $-0.30 \pm 0.02$ & \pm 0.13 & \pm 0.06 & +0.1 & $-0.10 \pm 0.00$ & \pm 0.06 & $+0.16 \pm 0.13$ & This paper \\
\hline $\mathrm{Be} 29$ & 4.0 & 22.00 & $-0.31 \pm 0.03$ & $+0.03 \pm 0.06$ & $+0.10 \pm 0.04$ & $+0.08 \pm 0.16$ & $-0.08 \pm 0.06$ & $-0.03 \pm 0.07$ & $+0.43 \pm 0.06$ & This paper \\
\hline
\end{tabular}

Figure 11 shows the radial gradients for $\alpha$-elements, Fe-peak elements and the $s$-process element $\mathrm{Ba}$, based on our sample and the other high resolution data from the literature. The plots indicate some amount of dispersion at any given Galactocentric distance (this is especially true for $\mathrm{Ba}$ ), with no obvious trends, with the only exception of $\mathrm{Ca}$ : in this case there seems to be a gradient with a positive slope $(0.08 \pm 0.02$, with correlation coefficient 0.64 ) in the inner disk. The interpretation of the distributions of these elements is rather complex and it is strictly related to the role of SNeIa and SNeII in the Galactic chemical enrichment (see, e.g. Yong et al. 2005). The comparison between our data and Galactic chemical evolution models, planned for a future paper of the series, will be aimed to distinguish the main contribution in element production by different nucleosynthesis process.

Whereas we find nearly solar $[\mathrm{X} / \mathrm{Fe}]$ ( $\alpha$ and Fe-peak elements) in all the clusters, Yong et al. (2005) and Carraro et al. (2004) claim that the outer disk clusters Sau 1, Be 20 and Be 29 (the two latter in common with the present study) have an enhancement in the $\alpha$-elements. Note, however, that Yong et al. (2005) derive $[\mathrm{Si}+\mathrm{Ca} / \mathrm{Fe}] \sim+0.1$, i.e. values similar to those we find for some stars, and which are not much higher than solar, and have been also found in field stars of similar metallicity; on the other hand, they derive $[\mathrm{Mg}+\mathrm{Ti} / \mathrm{Fe}] \sim+0.2-0.3$. Also the 

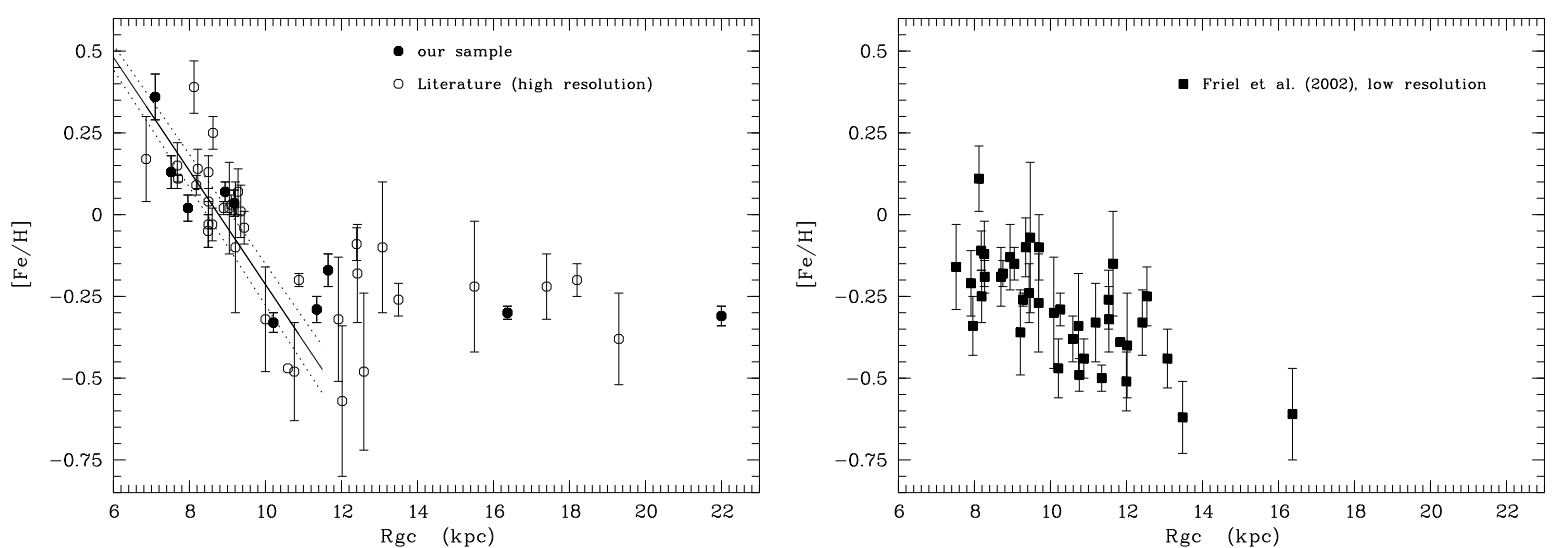

Fig. 9. Radial gradient ([Fe/H] vs. Galactocentric distance) for open clusters. Left panel: the results for clusters in our sample analyzed so far (filled circles; this paper, Papers I, II, and S07) are compared to other clusters analyzed with high-resolution spectroscopy (open circles). Right panel: the low resolution sample by Friel et al. (2002).
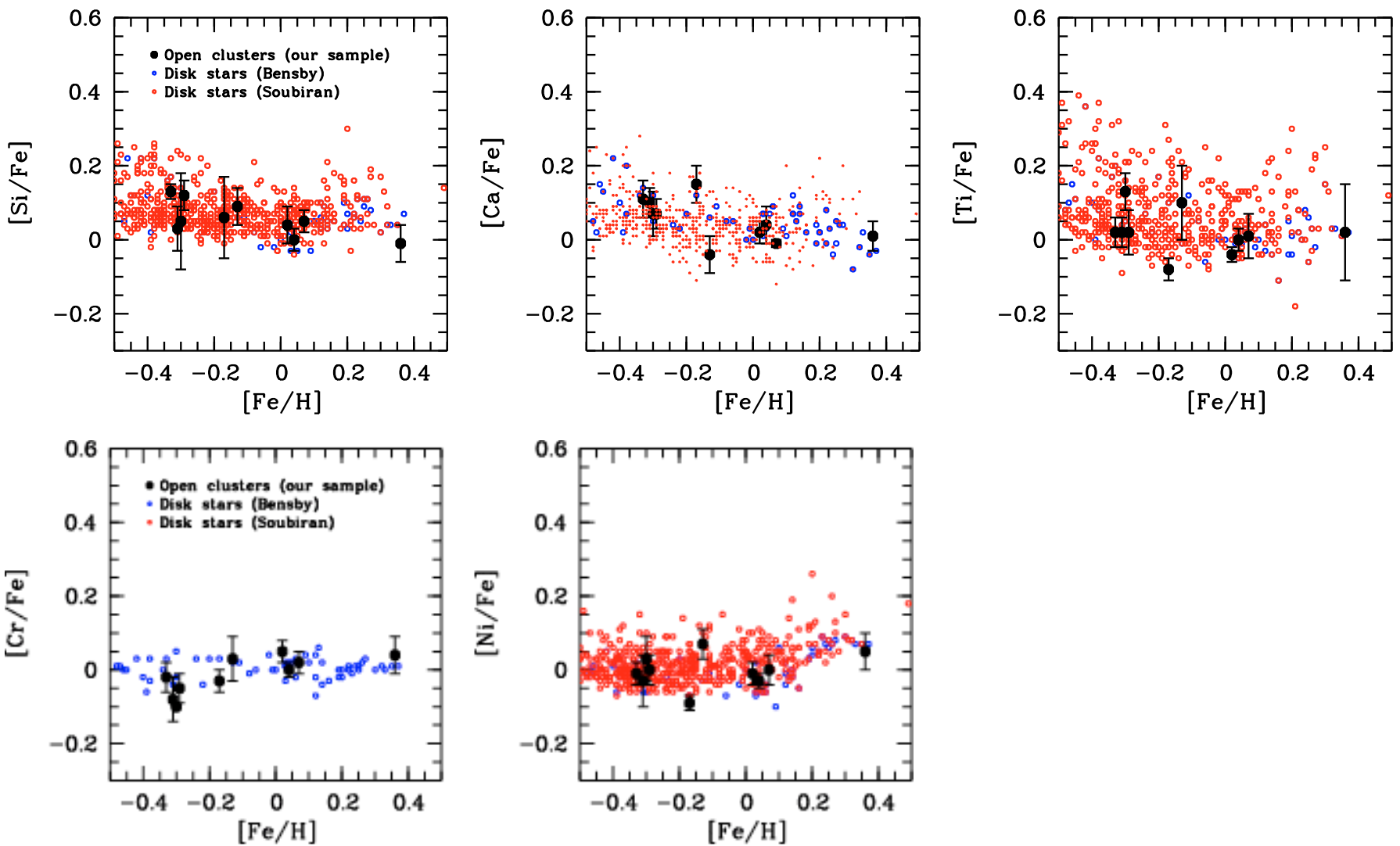

Fig. 10. $[\mathrm{X} / \mathrm{Fe}]$ vs. $[\mathrm{Fe} / \mathrm{H}]$ : comparison between open clusters in our sample (black filled circles) and disk stars (dots) by Bensby et al. (2005, blue) and Soubiran \& Girard (2005, red).

values for the $\alpha$-elements found by Carraro et al. (2004) are actually only slightly oversolar (average $[\alpha / \mathrm{Fe}]$ ratios of less than 0.1 for Be 29 and about 0.15 for Sau 1); anyway, in the most recent analysis of five outer disk clusters Carraro et al. (2007) do seem to converge on solar-scaled $[\alpha / \mathrm{Fe}]$ ratios, similar to open clusters in the solar vicinity and thin disk stars. With the present data, we do not think there is a strong argument for a significantly increased $[\alpha / \mathrm{Fe}]$ at large Galactocentric distances.

The only s-process element analyzed by us is $\mathrm{Ba}$, which is clearly enhanced for our open clusters. At the Fe abundances of our clusters, the Ba production is dominated by the $s$-processes in low mass stars (see e.g. Travaglio et al. 2001; and
Cescutti et al. 2006, for a complete discussion about this element). The Ba enhancement, mainly in the younger clusters, might be explained by its high production in low mass stars, mainly from 1 to $2 M_{\odot}$, that were formed from $\sim 7$ to 2 Gyr ago (e.g. Charbonnel et al. 1996). These stars contribute to the Ba enrichment exactly in the metallicity range covered by the examined clusters, while at lower metallicities the main contribution is from $r$-processes which are less effective (see also Fig. 4 by Cescutti et al. 2006). However, $\mathrm{Ba}$ abundances should be treated with caution, since the hyperfine structure could be important, as a preliminary analysis on available data seems to show (D'Orazi \& Randich, in preparation), contrary to what found e.g., by 

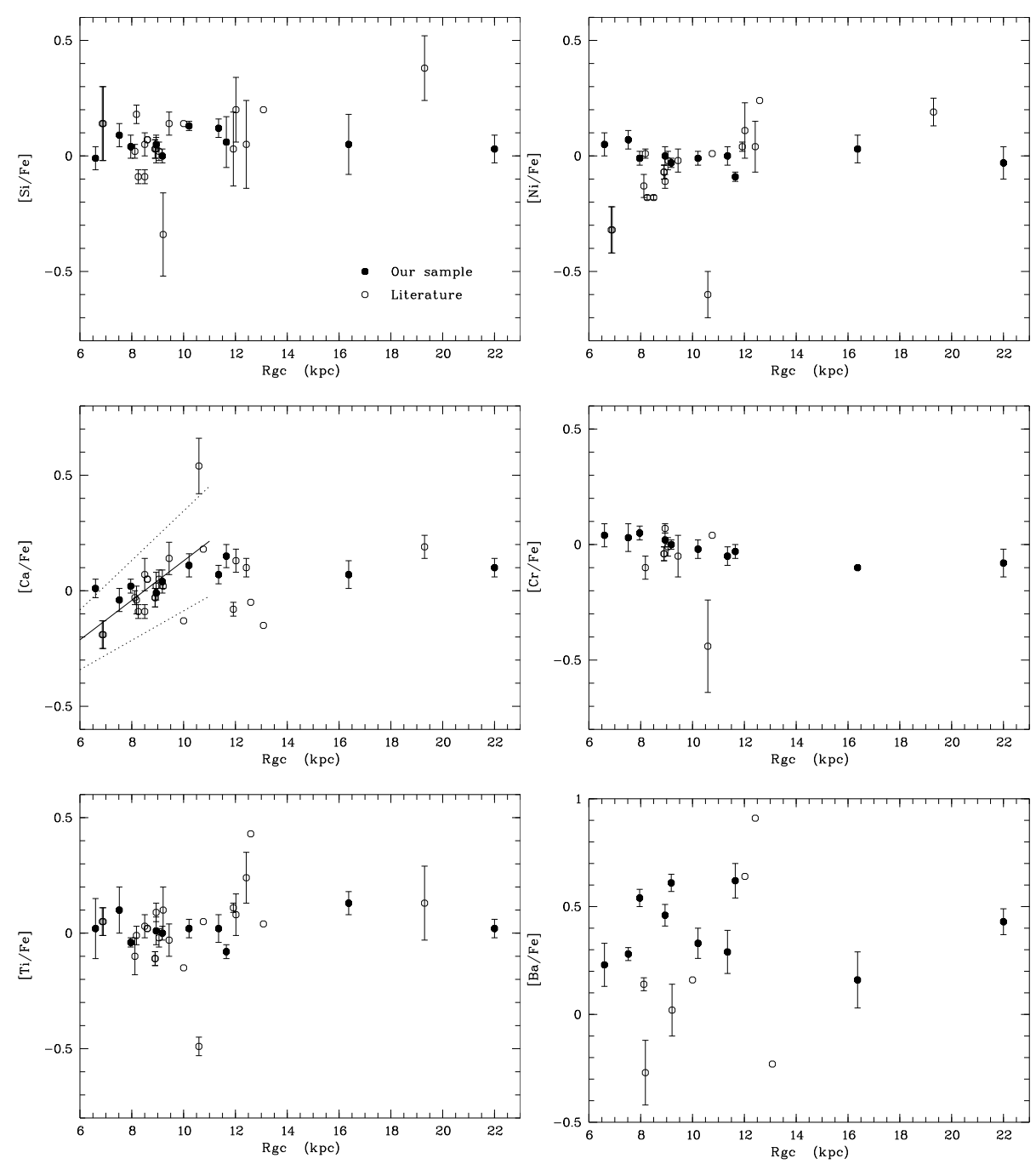

Fig. 11. Radial gradients of $\mathrm{Si}, \mathrm{Ca}, \mathrm{Ti}, \mathrm{Cr}, \mathrm{Ni}$, and $\mathrm{Ba}$, based on high resolution data: our sample (filled circles), and other literature investigations (open circles).

Mashonkina \& Gehren (2001); this would decrease the abundances. Furthermore, Ba abundances (ours and literature ones) show a scatter much higher than for any other element here considered, suggesting that we need a careful appraisal of their reliability before attempting meaningful interpretations.

\section{Summary}

This study is part of a project on the metallicity and chemical abundances of giant stars in open clusters, whose final goal is the determination of radial gradients in the Milky Way based on a statistically significant sample.

We report here on the abundances of Berkeley 20, Berkeley 29, Melotte 66, and Collinder 261; in particular, the first two clusters are outer disk objects, and the last is inside the solar circle, so they are critical for understanding the behavior of radial gradients.

- We derive subsolar $\mathrm{Fe}$ content $([\mathrm{Fe} / \mathrm{H}] \sim-0.30)$ for Be 20, Be 29 and Mel 66, and a slightly oversolar metallicity $(+0.13)$ for $\mathrm{Cr} 261$.

- The abundances of $\alpha$-elements ( $\mathrm{Si}, \mathrm{Ca}, \mathrm{Ti}$ ) and Fe-peak elements $(\mathrm{Cr}, \mathrm{Ni})$ are close to solar for most of the stars, with a small dispersion from the average in certain cases. The $s$-process element $\mathrm{Ba}$ is enhanced for all our samples, as often found for open clusters (but see the warning in last section).

- The abundances of $\mathrm{Mg}$ and $\mathrm{Al}$ have been derived from a few lines, which in general suggest oversolar values with respect to $\mathrm{Fe}$.

- Na abundances were computed both using EWs and LTE assumptions - in this case $[\mathrm{Na} / \mathrm{Fe}]$ values appear to be enhanced - and deriving non-LTE Na abundances using a lineprofile fitting technique, finding abundance ratios close to solar. This supports the idea that the claim of an enhancement in $\mathrm{Na}$ for evolved stars in open clusters might be due to neglected non-LTE effects for spectral lines, or to a non-adequate treatment of these effects. Further studies are needed to settle this issue.

The results for the element ratios of all the clusters in our sample have been compared to those of disk stars, finding a very good agreement: the $[\mathrm{X} / \mathrm{Fe}]$ abundances vs. $[\mathrm{Fe} / \mathrm{H}]$ of open clusters and disk stars are similar, i.e. the distributions are flat and close to solar, with the $\alpha$-elements, Ti in particular, showing a larger dispersion than Fe-peak elements.

We have combined our metallicities with literature ones and we find evidence for a steep negative slope of the radial metallicity gradient up to a radius of $\sim 10-11 \mathrm{kpc}$, while we confirm the flattening of the gradient in the outer disk. The radial 
distributions of our sample show for other elements a rather large amount of scatter without obvious trends with distance (with the possible exception of $\mathrm{Ca}$ ). The interpretation of the distributions of $\alpha$ - and Fe-peak elements is rather complex and it is strictly related to the role of Supernovae in the Galactic chemical enrichment. Our observational results can be used to distinguish the main contributions in element production by different nucleosynthesis processes, such as $\mathrm{SNe}$ Ia and $\mathrm{SNe}$ II. This will be the subject of a forthcoming paper.

Acknowledgements. P.S. acknowledges support by the Italian MIUR, under PRIN 20040228979-001. We are grateful to L. Prisinzano and P. Spanò for helpful discussion on cluster photometry and to P. Montegriffo for his useful software. This research has made use of the WEBDA database operated at the Institute for Astronomy of the University of Vienna and of NASA's Astrophysics Data System.

\section{References}

Allende Prieto, C., Asplund, M., \& Fabiani Bendicho, P. 2004, A\&A, 423, 1109 Alonso, A., Arribas, S., \& Martínez-Roger, C. 1999, A\&AS, 140, 261 Andreuzzi, G., Bragaglia, A., \& Tosi, M. 2007, in XXI Century challenges for stellar evolution, ed. S. Cassisi, \& M. Salaris, in press, MemSAIt, 79

Andreuzzi, G., Bragaglia, A., \& Tosi, M. 2008, MNRAS, submitted Andrievsky, S. M., Spite, M., Korotin, S. A., et al. 2007, A\&A, 464, 1081 Bensby, T., Feltzing, S., Lundström, I., \& Ilyin, I. 2005, A\&A, 433, 185 Bessell, M. S. 1979, PASP, 91, 589

Bragaglia, A., \& Tosi, M. 2006, AJ, 131, 1544

Bragaglia, A., Carretta, E., Gratton, R. G., et al. 2001, AJ, 121, 327

Bragaglia, A., Held, E. V., \& Tosi, M. 2005, A\&A, 429,

Bragaglia, A., Tosi, M., Carretta, E., et al. 2006, MNRAS, 366, 1493

Bragaglia, A., Sestito, P., Villanova, S., et al. 2008, A\&A, 480, 79 (Paper II)

Brown, J. A., Wallerstein, G., Geisler, D., \& Oke, J. B. 1996, AJ, 112, 1551

Carlsson, M. 1986, Uppsala Obs. Rep., 33

Carraro, G., Ng, Y. K., \& Portinari, L. 1998, MNRAS, 296, 1045

Carraro, G., Bresolin, F., Villanova, S., et al. 2004, AJ, 128, 1676

Carraro, G., Janes, K. A., Costa, E., \& Méndez, R. A. 2006a, MNRAS, 368 , 1078

Carraro, G., Villanova, S., Demarque, P., et al. 2006b, ApJ, 643, 1151

Carraro, G., Geisler, D., Villanova, S., Frinchaboy, P., \& Majewski, S. 2007, A\&A, 476, 217

Carretta, E., Bragaglia, A., Gratton, R., \& Tosi, M. 2004, A\&A, 422, 951

Carretta, E., Bragaglia, A., Gratton, R., \& Tosi, M. 2005, A\&A, 441, 131

Cescutti, G., Francois, P., Matteucci, F., Cayrel, R., \& Spite, M. 2006, A\&A, 448,557

Charbonnel, C., Meynet, G., Maeder, A., \& Schaerer, D. 1996, A\&AS, 115, 339

Corder, S., \& Twarog, B. A. 2001, AJ, 122, 895

Feltzing, S., \& Gustafsson, B. 1998, A\&AS, 129, 237

Fitzpatrick, M. J., \& Sneden, C. 1987, BAAS, 19, 1129

Ford, A., Jeffries, R. D., \& Smalley, B. 2005, MNRAS, 364, 272

Freeman, K., \& Bland-Hawthorn, J. 2002, ARA\&A, 40, 487

Friel, E. D. 1995, ARA\&A, 33, 381

Friel, E. D. 2006, in Chemical Abundances and Mixing in Stars in the Milky Way and its Satellites, ed. S. Randich, \& L. Pasquini, ESO Astrophys. Symp., 24, 3

Friel, E. D., Janes, K. A., Tavarez, M., et al. 2002, AJ, 124, 2693

Friel, E. D., Jacobson, H. R., Barrett, E., et al. 2003, AJ, 126, 2372

Friel, E. D., Jacobson, H. R., \& Pilachowski, C. A. 2005, AJ, 129, 272

Frinchaboy, P. M., Munöz, R. R., Phleps, R. L., Majewski, S. R., \& Kunkel, W. E. 2006, AJ, 131, 922

Froebrich, D., Scholz, A., \& Raftery, C. L. 2007, MNRAS, 374, 399
Gonzalez, G., \& Wallerstein, G. 2000, PASP, 112, 1081

Gozzoli, E., Tosi, M., Marconi, G., \& Bragaglia, A. 1996, MNRAS, 283, 66 Gratton, R. G., \& Contarini, G. 1994, A\&A, 283, 911

Gratton, R., Carretta, E., Eriksson, K., \& Gustafsson, B. 1999, A\&A, 350, 955

Gratton, R., Sneden, C., \& Carretta, E. 2004, ARA\&A, 42, 385

Gratton, R., Bragaglia, A., Carretta, E., \& Tosi, M. 2006, ApJ, 642, 462

Hamdani, S., North, P., Mowlavi, N., Raboud, D., \& Mermilliod, J.-C. 2000, A\&A, 360, 509

Jacobson, H. R., Friel, E. D., \& Pilachowski, C. A. 2007, AJ, 134, 1216

Janes, K. A., \& Phelps, R. L. 1994, AJ, 108, 1773

Kaluzny, J. 1994, A\&AS, 108, 151

Kassis, M., Janes, K. A., Friel, E. D., \& Phelps, R. L. 1997, AJ, 113, 1723

Korotin, S. A., Andrievsky, S. M., \& Luck, R. E. 1999a, A\&A, 351, 168

Korotin, S. A., Andrievsky, S. M., \& Kostynchuk, L. Yu. 1999b, Ap\&SS, 260, 531

Kronberger, M., Teutsch, P., Alessi, B., et al. 2006, A\&A, 447, 921

Kurucz, R. L. 1993, CD-ROM No. 9

MacMinn, D., Phelps, R. L., Janes, K. A., \& Friel, E. D. 1994, AJ, 107, 1806

Mashonkina, L. I., \& Gehren, T. 2001, A\&A, 376, 232

Mashonkina, L. I., Shimanskii, V. V., \& Sakhibullin, N. A. 2000, Astron. Rep., 44, 790

Mazur, B., Krzeminski, W., \& Kaluzny, J. 1995, MNRAS, 273, 59

Mishenina, T. V., Bienaymé, O., Gorbaneva, T. I., et al. 2006, A\&A, 456, 1109

Origlia, L., Valenti, E., Rich, M. R., \& Ferraro, F. R. 2006, ApJ, 646, 499

Ortolani, S., Bica, E., \& Barbuy, B. 2005, A\&A, 437, 531

Pallavicini, R., Spanò, P., Prisinzano, L., Randich, S., \& Sestito, P. 2006, in Chemical Abundances and Mixing in Stars in the Milky Way and its Satellites, ed. S. Randich, \& L. Pasquini, ESO Astrophys. Symp., 24, 181

Pasquini, L., Avila, G., Allaert, E., et al. 2000, SPIE, 4008, 129

Pasquini, L., Randich, S., Zoccali, M., et al. 2004, A\&A, 424, 951

Paulson, D. B., Sneden, C., \& Cochran, W. D. 2003, AJ, 125, 3185

Randich, S., Pallavicini, R., Meola, G., Stauffer, J. R., \& Balachandran, S. C. 2001, A\&A, 372, 862

Randich, S., Sestito, P., \& Pallavicini, R. 2003, A\&A, 399, 133

Randich, S., Bragaglia, A., Pastori, L., et al. 2005, ESO Messenger, 121, 18

Randich, S., Sestito, P., Primas, F., Pallavicini, R., \& Pasquini, L. 2006, A\&A, 450,557

Schuler, S. C., King, J. R., Fischer, D. A., Soderblom, D. R., \& Jones, B. F. 2003, AJ, 125, 2085

Schuler, S. C., King, J. R., Hobbs, L. M., \& Pinsonneault, M. H. 2004, ApJ, 602, 117

Sestito, P., Randich, S., Mermilliod, J.-C., \& Pallavicini, R. 2003, A\&A, 407, 289

Sestito, P., Bragaglia, A., Randich, S., et al. 2006, A\&A, 458, 121 (Paper I)

Sestito, P., Randich, S., \& Bragaglia, A. 2007, A\&A, 465, 185 (S07)

Smith, V. V., \& Suntzeff, N. B. 1987, AJ, 93, 359

Sneden, C. A. 1973, ApJ, 184, 839

Soubiran, C., \& Girard, P. 2005, A\&A, 139, 151

Spanò, P. 2005, Università degli Studi di Palermo, Ph.D. Thesis

Skrutskie, M. F., Cutri, R. M., Stiening, R., et al. 2996, AJ, 131, 1163

Tautvaisiene, G., Edvardsson, B., Puzeras, E., \& Ilyin, I. 2005, A\&A, 431, 933

Tosi, M. 1996, in From Stars to Galaxies: the Impact of Stellar Physics on Galaxy Evolution, ed. C. Leitherer, U. Fritze-von-Alvensleben, \& J. Huchra, ASPC, 98, 299

Tosi, M., Di Fabrizio, L., Bragaglia, A., Carusillo, P. A., \& Marconi, G. 2004, MNRAS, 354, 225

Tosi, M., Bragaglia, A., \& Cignoni, M. 2007, MNRAS, 378, 730

Travaglio, C., Galli, D., \& Burkert, A. 2001, ApJ, 547, 217

Twarog, B. A., Anthony-Twarog, B. J., \& Hawarden, T. G. 1995, PASP, 107, 1215

Twarog, B. A., Ashman, K. M., \& Anthony-Twarog, B. J. 1997, AJ, 114, 2556

Villanova, S., Carraro, G., Bresolin, F., \& Patat, F. 2005, AJ, 130, 652

Yong, D., Carney, B. W., \& De Almeida, L. 2005, AJ, 130, 597 